\title{
Oligodendrogliogenic and neurogenic adult subependymal zone neural stem cells constitute distinct lineages and exhibit differential responsiveness to Wnt signalling
}

Felipe Ortega ${ }^{1,2,8}$, Sergio Gascón ${ }^{1,3,7}$, Giacomo Masserdotti ${ }^{1,3,7}$, Aditi Deshpande ${ }^{1}$, Christiane Simon ${ }^{1}$, Judith Fischer ${ }^{3}$, Leda Dimou ${ }^{1,3}$, D. Chichung Lie ${ }^{4}$, Timm Schroeder ${ }^{5}$ and Benedikt Berninger ${ }^{1,2,3,6,8}$

The adult mouse subependymal zone (SEZ) harbours adult neural stem cells (aNSCs) that give rise to neuronal and oligodendroglial progeny. However it is not known whether the same aNSC can give rise to neuronal and oligodendroglial progeny or whether these distinct progenies constitute entirely separate lineages. Continuous live imaging and single-cell tracking of aNSCs and their progeny isolated from the mouse SEZ revealed that aNSCs exclusively generate oligodendroglia or neurons, but never both within a single lineage. Moreover, activation of canonical Wnt signalling selectively stimulated proliferation within the oligodendrogliogenic lineage, resulting in a massive increase in oligodendrogliogenesis without changing lineage choice or proliferation within neurogenic clones. In vivo activation or inhibition of canonical Wnt signalling respectively increased or decreased the number of Olig2 and PDGFR- $\alpha$ positive cells, suggesting that this pathway contributes to the fine tuning of oligodendrogliogenesis in the adult SEZ.

The adult subependymal zone (SEZ) constitutes a niche of life-long neuro- and oligodendrogliogenesis in most mammalian species ${ }^{1}$, with neuronal progeny being destined for the olfactory bulb², whereas new oligodendroglia migrates towards the corpus callosum and the white matter tracts of the striatum and the fimbria fornix, under both physiological conditions $s^{3-5}$ and demyelinating pathologies ${ }^{6}$. Both neuronal and oligodendroglial progeny are believed to arise from aNSCs of astro-/radial glial identity $4,7,8$. However, there is accruing evidence that the adult SEZ is highly regionalized, with neuronal progeny of distinct identity being generated at different areas along the dorsoventral and rostrocaudal axes ${ }^{5,9-11}$. This mosaic organization of the adult SEZ may reflect the regionalization of the forebrain during development ${ }^{12}$ and suggests that aNSCs, although sharing astro-/radial glial characteristics ${ }^{3,13}$, may not have the potential to generate all types of neuronal progeny under physiological conditions.

This begs the question of whether oligodendrogliogenesis is equally regionalized and whether one and the same aNSC can give rise to neuronal and oligodendroglial progeny. Pathologies such as demyelinating diseases or genetic manipulations altering bone morphogenic protein (BMP) signalling have strongly suggested that neuro- and oligodendrogliogenesis are indeed competing cell fates ${ }^{14,15}$. In both instances, the oligodendrogliogenic transcription factor Olig2 was upregulated, resulting in the suppression of a neurogenic fate ${ }^{14}$ or even conversion of cells already committed to a neuronal identity ${ }^{15}$. Nonetheless, whether NSCs can give rise to both lineages under physiological conditions remains an unresolved question.

We have recently used a primary culture of the adult SEZ to study the lineage progression of aNSCs by continuous live imaging ${ }^{16,17}$. Adult NSCs in these cultures were characterized by a prolonged cell cycle and marked cell growth before division when compared with transit-amplifying precursors (TAPs). Moreover, these cells exhibit an active glial fibrillary acidic protein (GFAP) promoter characteristic of their astro-/radial glial identity $7,13,18,19$. Finally, aNSCs derived from the ventrolateral aspect of the SEZ gave rise to asymmetric lineage

\footnotetext{
${ }^{1}$ Department of Physiological Genomics, Institute of Physiology, Ludwig-Maximilians University Munich, Schillerstrasse 46 , D-80336 Munich, Germany. ${ }^{2}$ Institute of Physiological Chemistry, University Medical Center Johannes Gutenberg University, Hanns-Dieter-Hüsch-Weg 19, D-55128 Mainz, Germany. ${ }^{3}$ Institute of Stem Cell Research, Helmholtz Zentrum München, Ingolstädter Landstrasse 1, D-85764 Neuherberg, Germany. ${ }^{4}$ Institute of Biochemistry, Emil Fischer Center, University

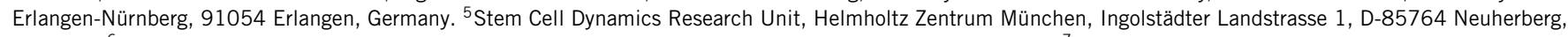
Germany. ${ }^{6}$ Focus Program Translational Neuroscience, Johannes Gutenberg University, 55131 Mainz, Germany. ${ }^{7}$ These authors contributed equally to this work. ${ }^{8}$ Correspondence should be addressed to F.O. or B.B. (e-mail: ortegaf@uni-mainz.de or berningb@uni-mainz.de)
} 
trees consisting of both neuronal and astroglial progeny ${ }^{17}$. However, in these cultures we encountered very few oligodendroglial cells ${ }^{16}$, consistent with the overall scarce production of oligodendroglia in the ventrolateral SEZ (refs 4,5).

In the present study we show that oligodendrogliogenic aNSCs are enriched in the dorsal aspect of the SEZ. Continuous live imaging in vitro revealed that neuro- and oligodendrogliogenic lineages arise from aNSCs exhibiting a protracted cell cycle and an active GFAP promoter. However, we never observed the genesis of neurons and oligodendroglia within the same lineage tree. Moreover, we found that the oligodendrogliogenic lineage is selectively responsive to canonical Wnt signalling, known to be particularly prominent in the dorsal SEZ in $v i v o^{20}$.

\section{RESULTS}

\section{Oligodendroglial progeny is enriched in primary cultures containing the dorsal SEZ}

Although the adult SEZ is known to serve as a source for new oligodendroglial cells ${ }^{3}$, in previous studies we observed rather low numbers of oligodendroglial progeny in cultures of aNSCs isolated from the lateral SEZ. However, we noticed that cultures containing cells derived from both the dorsal and the lateral aspect of the SEZ contained considerably more NG2-immunoreactive oligodendroglial cells $^{21}$ (Fig. 1a-c; NG2 is also known as CSPG4, chondroitin sulfate proteoglycan 4). This suggests that the dorsal SEZ has a higher rate of oligodendrogliogenesis than the lateral SEZ. Consistent with this interpretation, 7 days following retroviral birth-dating in vivo we observed more progeny exhibiting an oligodendroglial morphology or expressing the oligodendrocyte progenitor marker PDGFR $\alpha$ (ref. 22) in the dorsal part than the lateral (Fig. 1d and Supplementary Fig. S1a). Likewise, we noted a significant difference in the number of green fluorescent protein (GFP)- and NG2-double-positive cells between these two SEZ areas in mice expressing a tamoxifen-inducible Cre recombinase under the oligodendroglial lineage-specific Sox 10 promoter, crossed to a reporter

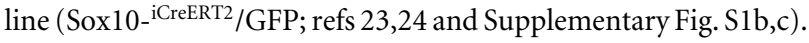

\section{Neuronal and oligodendroglial progeny arise from distinct lineages}

Although aNSCs have been proposed to give rise to all major neural lineages, concomitant neuro- and oligodendrogliogenesis from single aNSCs has not been observed directly. To address this question we carried out continuous live imaging of primary cultures of the dorsal and lateral adult SEZ to track the lineage progression of aNSCs (refs 16,17). In this culture aNSCs can be identified by a slow cell cycle, substantial cell growth before division and asymmetric cell division within the lineage tree ${ }^{16}$. We encountered lineage trees originating from aNSCs fulfilling the above criteria and generating large clones of $\beta$ III-tubulin-positive neurons and few GFAP-positive astroglia (Fig. 2a and Supplementary Fig. S2a). In these neurogenic clones none of the progeny was positive for NG2 (Fig. 2a and Supplementary Fig. S2a). In contrast, few founder cells gave rise to NG2-positive oligodendroglia (Fig. 2b-d and Supplementary Fig. S3a and Video S1). However, these clones never contained any $\beta$ III-tubulin-positive progeny (Fig. $2 \mathrm{~b}-\mathrm{d}$ and Supplementary Fig. S3a). We also observed an instance of asymmetric cell division in which the founder cell gave rise to both NG2-positive oligodendroglial and GFAP-positive astroglial progeny
(Fig. 2d and Supplementary Fig. S4a). Many of the clonal founder cells were characterized by a slow cell cycle (Fig. 2e) and exhibited massive cell growth before division (Fig. 2f). Moreover, using a mouse line in which monomeric red fluorescent protein 1 (mRFP1) is driven by the hGFAP promoter ${ }^{25}$ we observed the generation of oligodendrogliogenic clones from slow-dividing mRFP1-positive cells (Supplementary Fig. S4b). None of the cells that expressed mRFP1 for the first $24 \mathrm{~h}$ following plating was found to co-express NG2, indicating that the cells that give rise to oligodendrogliogenic clones are not oligodendrocyte precursor cells (OPCs) right from the beginning, but rather aNSCs (Supplementary Fig. S2d). Finally, clones containing both NG2- and GFAP-positive progeny from GFP-reporter positive cells could be observed following fate mapping using the GLAST :: CreERT2/GFP mouse line ${ }^{24,26}$ (Fig. 2g). These data indicate that the SEZ harbours aNSCs that are capable of, and restricted to, generating NG2-positive oligodendroglial and GFAP-positive astroglial progeny. Strikingly, in no instance ( $n>110$ clones) did we observe the generation of both neuronal and oligodendroglial progeny from a single clonal founder cell, strongly supporting the notion that these two lineages remain strictly separated. To further characterize the progenitors giving rise to the neuro- or oligodendrogliogenic lineages we used SEZ cultures isolated from hGFAP-RFP mice and analysed the expression of different fate determinants $24 \mathrm{~h}$ after plating. We observed that $18 \pm 1 \%$ of the RFPpositive cells expressed Olig2, a marker of the oligodendrogliogenic lineage, whereas $65 \pm 12 \%$ expressed Pax6, indicative of commitment to the neuronal lineage. Finally, $75 \pm 5 \%$ of the RFP-positive cells were immunoreactive to Mash1, known to be expressed in both lineages. Given that $85 \%$ of the clones are neurogenic and $9 \%$ of the clones are oligodendrogliogenic, with the remaining $6 \%$ consisting of GFAP-positive cells only, these data suggest that the expression of Pax6 and Olig2 is indicative of a neuronal and oligodendroglial fate commitment as early as the aNSC stage (Supplementary Fig. S2c-e).

\section{Wnt signalling increases oligodendroglial progeny in primary cultures of the adult mouse SEZ}

Given that canonical Wnt signalling is strongly activated in the dorsal SEZ (ref. 20), we hypothesized that Wnt signalling may contribute to the regulation of adult SEZ oligodendrogliogenesis. To assess this we first treated primary cultures of the adult SEZ (dorsal + lateral) with recombinant Wnt3a protein, which is known to activate the canonical Wnt signalling pathway ${ }^{27}$. After 6 days of continuous treatment, the number of NG2-positive cells increased eightfold (Fig. 3a,b). This effect was highly specific to the oligodendroglial lineage, as the number of GFAP-positive astroglia and $\beta$-III-tubulin-positive neurons remained unchanged (Fig. 3a,b). This lineage-specific effect of Wnt3a stands in striking contrast to the effect of growth factors EGF (refs 3,16,28) and FGF2 (Fig. 3e,f), which concomitantly increase both oligodendroglial and astroglial progenies. The specificity of the Wnt3a effect on the oligodendroglial lineage was further corroborated by the selective increase in oligodendroglial messenger RNA transcripts, including the OPCspecific cell cycle regulator cyclin D1 (ref. 29), but not astroglial or neuronal mRNAs, with the notable exception of NeuroD1 and Eomes (Tbr2; Fig. 3d and Supplementary Table S2). The effect of Wnt3a was mainly mediated by the canonical signalling pathway, because it was markedly reduced in the presence of the canonical Wnt signalling inhibitor dickkopf homolog 1 (DKK1; ref. 30; Fig. 3c). Conversely, treatment 
a

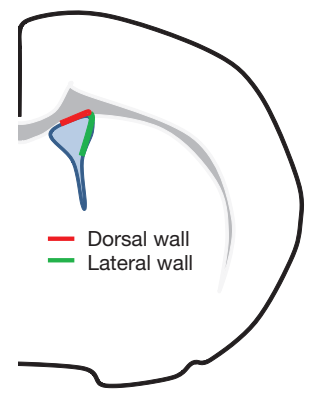

c

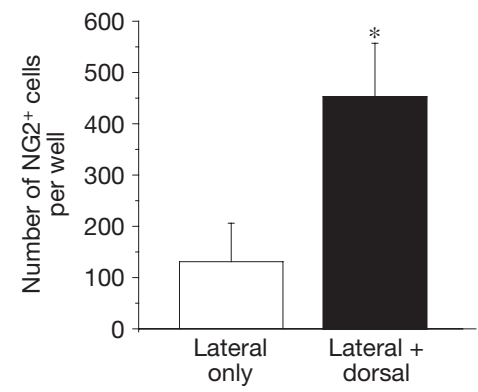

b

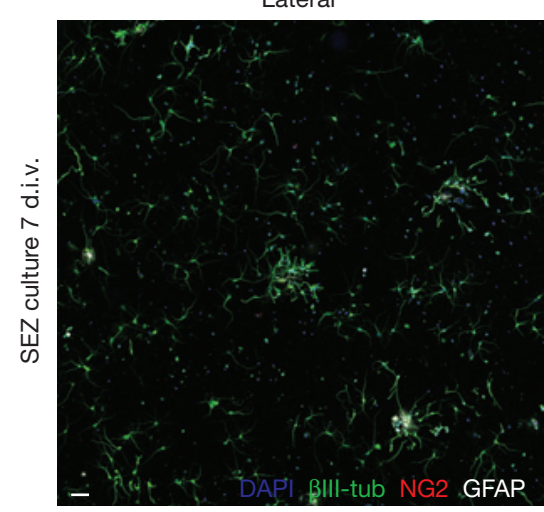

d

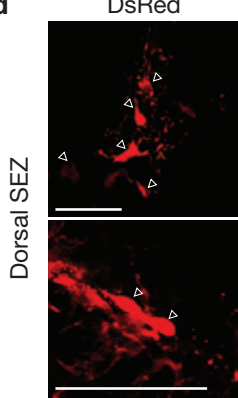

DsRed

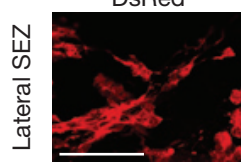

PDGFR $\alpha$

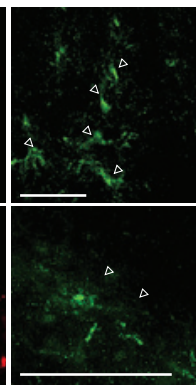

PDGFR $\alpha$

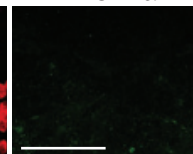

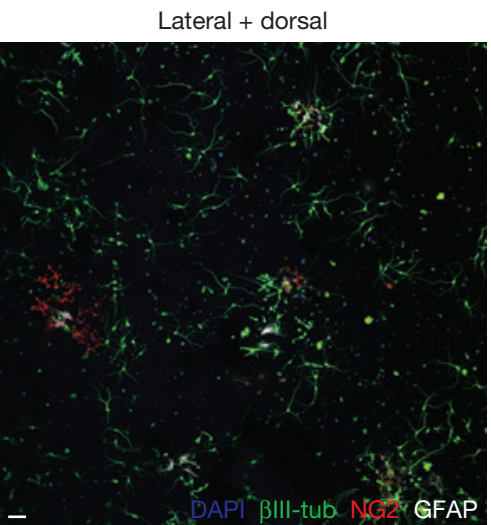

DCX

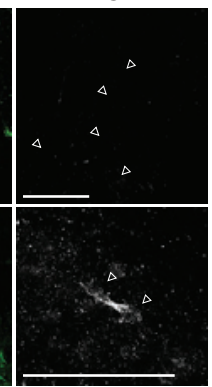

$\mathrm{DCX}$

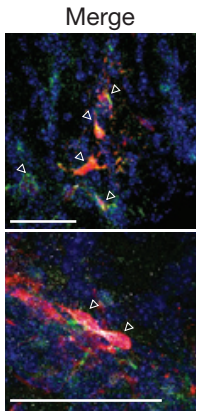

Merge
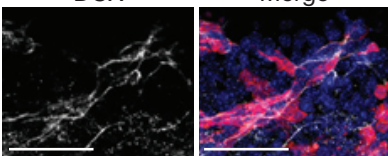

Figure 1 Oligodendroglial progenitors are enriched within the dorsal wall of the adult SEZ. (a) A schematic diagram demarcating the dorsal and lateral walls of the adult SEZ. (b) NG2-positive (red) oligodendroglia, BIII-tubulin-positive (green) neuroblasts and GFAP-positive (white) astroglia in cultures of the lateral wall and of the lateral plus dorsal walls of the adult SEZ, respectively; blue, DAPI. d.i.v., days in vitro. (c) Quantification of

with Wnt7a protein, an activator of the non-canonical signalling pathway ${ }^{27}$, was not able to reproduce the effect of Wnt3a (Fig. 3c).

The effect of Wnt3a treatment was abolished by co-treatment with recombinant BMP4 (Fig. 3g), consistent with a previous report that BMP signalling in the lateral SEZ suppresses oligodendrogliogenesis in vivo ${ }^{14}$. Moreover, BMP4 treatment reduced the number of NG2positive cells in control cultures containing both the dorsal and lateral SEZ to levels similar to those in lateral SEZ-only cultures (362 BMP4-treated versus 1,273 control-treated cells per $339 \mathrm{~mm}^{2}$ area). Conversely, treatment of cultures of the lateral SEZ- only cultures with the BMP inhibitor noggin increased the number of NG2-positive cells (694 noggin-treated versus 318 control-treated cells per $339 \mathrm{~mm}^{2}$ area), suggesting that the low level of oligodendrogliogenesis in lateral SEZ-only cultures is due to endogenous BMP signalling ${ }^{14}$.

\section{Wnt stimulates proliferation by shortening cell cycle length}

This massive increase in NG2-positive cells following Wnt3a treatment could be accounted for by either the activation of quiescent aNSCs, an increase in proliferation within the lineage, an increase in survival or a fate switch of neurogenic aNSCs toward the oligodendroglial lineage. To discern between these possibilities we analysed the number of oligodendrogliogenic lineage trees, cell cycle length, number of the number of NG2-positive cells in lateral and lateral plus dorsal cultures ( $t$-test, ${ }^{*} P<0.05$, s.e.m. $n=8$ independent experiments). (d) Enrichment of oligodendroglial progenitors in the dorsal versus lateral wall of the adult SEZ in vivo. Progeny of retrovirus labelled cells (Discosoma red fluorescent protein, DsRed, red), positive for either PDGFR (oligodendroglia, green) or DCX (doublecortin; neuroblasts, white). Scale bars, $50 \mu \mathrm{m}$.

amplifying rounds of division and the cellular composition of each clone. Figure 4a and Supplementary Video S2 show an example of an oligodendrogliogenic lineage tree originating from a slow-dividing aNSC that was characterized by marked cell growth before cell division. In agreement with the subsequent acquisition of an oligodendroglial identity, mRFP1 expression became downregulated along lineage progression (Supplementary Video S2). Analysis of several experiments revealed a marked increase in the number of cell divisions within the time window of tracking (Fig. 4a,b; Supplementary Fig. S5b). In Wnt3atreated cultures, oligodendroglial lineage trees showed an average of $5.70 \pm 0.41$ rounds of division, compared with $3.25 \pm 0.46$ in control conditions ( $P<0.001$; Fig. $4 \mathrm{e}$ and Supplementary Fig. S3). Accordingly, cell cycle length was significantly decreased when compared with controls (Fig. 4d). We also observed asymmetric cell divisions within clones generated from mRFP1-positive founder cells generating both oligodendroglial and astroglial progeny (Fig. 4c and Supplementary Fig. S5a). Another salient feature of the Wnt3a effect was the enhanced migratory behaviour of cells within oligodendrogliogenic clones (Fig. 4f), including the founder cells (for example see the position of the founder cell between 0-00:05 and 1-15:30 in Fig. 4a and Supplementary Video S2). Finally, we noted that clonal founder cells exhibited massive cell growth before cell division (Fig. 4g). 


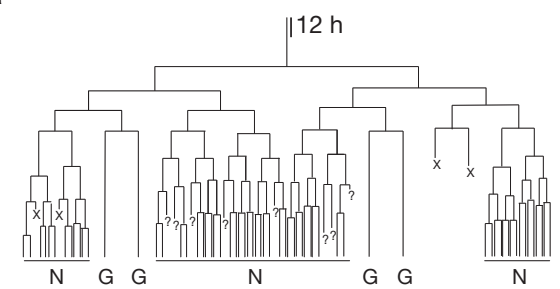

c

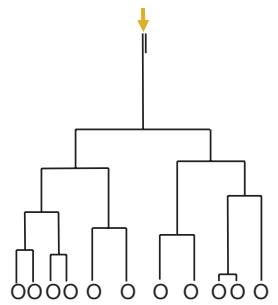

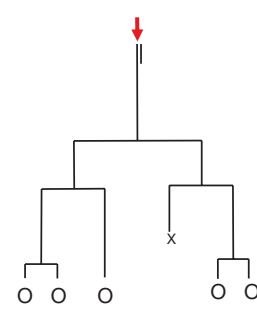

d

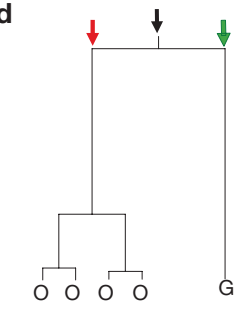

b
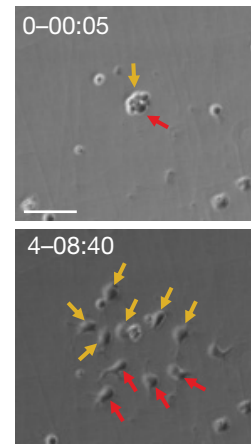

NG2
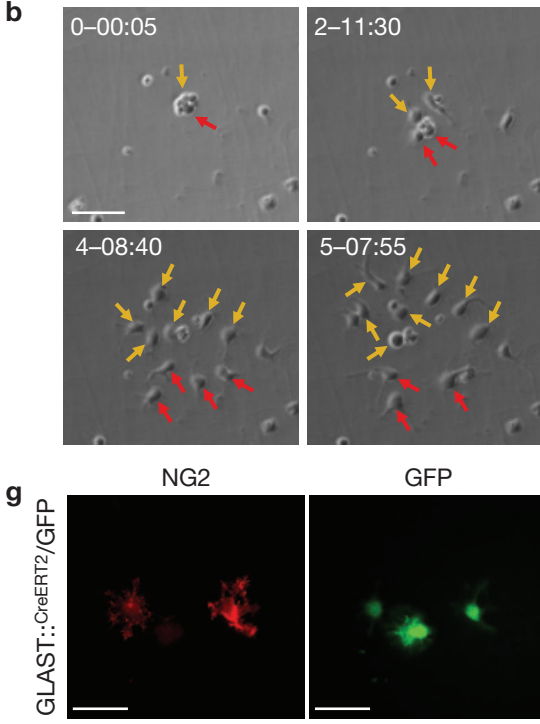

GFP
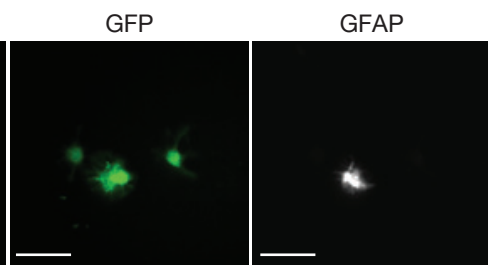

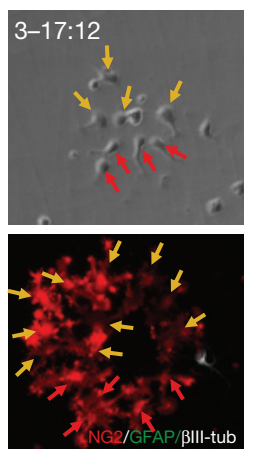

Merge

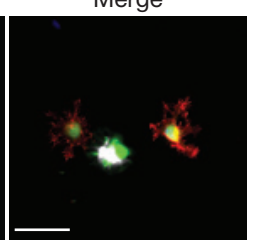

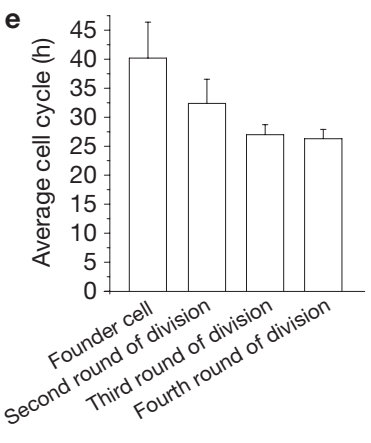

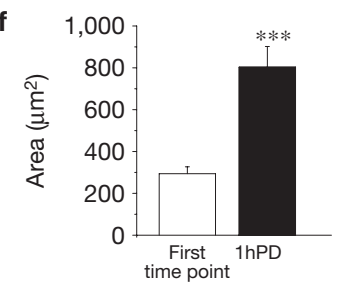

Figure 2 Neuro- and oligodendrogliogenic lineage trees tracked by live imaging in cultures of the dorsal plus lateral wall of the adult SEZ. (a) Asymmetric lineage tree generating neuronal and astroglial progeny (N, neuron; G, GFAP-positive astroglia; X, cell death; ?, cell could not be tracked till the end). (b) Phase contrast microscopy images obtained by time-lapse video microscopy at different times (day-hour:minute); last image post-imaging immunocytochemistry for NG2 (red), GFAP (green) and $\beta$ III-tubulin (white). (c) Symmetric lineage trees derived from the live imaging experiment depicted in $\mathbf{b}(\mathrm{O}$, oligodendroglia). Note the colour code of arrows for the respective clones. (d) Asymmetric lineage tree

We also noted a $40 \%$ increase in the number of oligodendrogliogenic clones (Supplementary Fig. S3), suggesting that Wnt3a treatment results in the activation of quiescent aNSCs or a switch in cell lineage. Consistent with the above finding that Wnt3a treatment did not alter the number of $\beta$ III-tubulin-positive neurons, we observed no changes in the lineage progression of neurogenic clones (Fig. $4 \mathrm{~h}$ and Supplementary Fig. S2b).

To distinguish between activation of quiescent aNSCs and a switch from neuronal to oligodendroglial fate, we first monitored adult SEZ cultures under control conditions and subsequently added Wnt3a. Whereas the addition of Wnt3a did not alter the division pattern and cell fate decisions within neurogenic clones (Fig. 5a,b) the same treatment clearly enhanced the rate of proliferation within the oligodendrogliogenic lineage by decreasing cell cycle length (Fig. 5a,c and Supplementary Video S3).

\section{Wnt signalling stimulates oligodendrogliogenesis in vivo}

Consistent with a role of canonical Wnt signalling in the adult SEZ in vivo, we could detect various components of the canonical Wnt signalling pathway (dishevelled 1, Lrp5 co-receptors and Wnt3 ligand). Moreover, these signalling components were differentially generating oligodendroglial and astroglial progeny. (e) Quantification of cell cycle length of all dividing cells tracked within oligodendrogliogenic lineage trees depending on the round of divisions. (f) Quantification of the cell size of the founder cells of oligodendrogliogenic clones at the start of the experiment and $1 \mathrm{~h}$ before division (1hPD) ( $t$-test, ${ }^{* * *} P<0.001$, s.e.m. $n=10$ cells obtained by gathering data of three independent experiments (variability represents an aggregation over three experiments)). (g) Fate-mapping analysis of oligodendrogliogenic clones using cultures

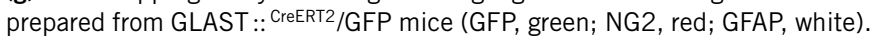
Scale bars, $50 \mu \mathrm{m}$.

expressed between the dorsal and the ventral wall of the SEZ, in agreement with the differences in oligodendrogliogenesis in these regions (Fig. 6e). Thus, we next addressed the question of whether canonical Wnt signalling augments oligodendrogliogenesis in the adult SEZ using a lentivirus for local expression of Wnt3 (LV-Wnt3-iresGFP; ref. 31). We confirmed in vitro that lentivirus-expressed Wnt 3 is indeed secreted from cells isolated from the adult SEZ and exerts a similar effect as recombinant Wnt3a protein (Supplementary Fig. S6). Next we proceeded to inject LV-Wnt3-iresGFP or a lentivirus expressing GFP only (LV-GFP) as control into the adult SEZ at lateral and dorsal locations. We counted the number of cells expressing Olig2 and PDGFR $\alpha$ within the area defined by GFP expression 14 days after lentivirus injection, and normalized it to the respective areas. Lentiviral expression of Wnt3 increased the number of Olig2- and PDGFR $\alpha$-positive cells significantly, in both the lateral and dorsal SEZ (Olig2-dorsal SEZ, $8590 \mathrm{Wnt3-treated} \mathrm{versus} 6451$ control-treated cells $\mathrm{mm}^{-2}$; lateral SEZ, $1985 \mathrm{Wnt} 3$-treated versus 946 control-treated cells $\mathrm{mm}^{-2}$; PDGFR $\alpha$-dorsal SEZ, 1367 Wnt3-treated versus 773 control-treated cells $\mathrm{mm}^{-2}$; lateral SEZ, 955 Wnt3-treated versus 424 control-treated cells $\mathrm{mm}^{-2}$; Fig. 6a-c). As in vitro, the effect of Wnt3 in the adult SEZ was restricted to the oligodendroglial 
a

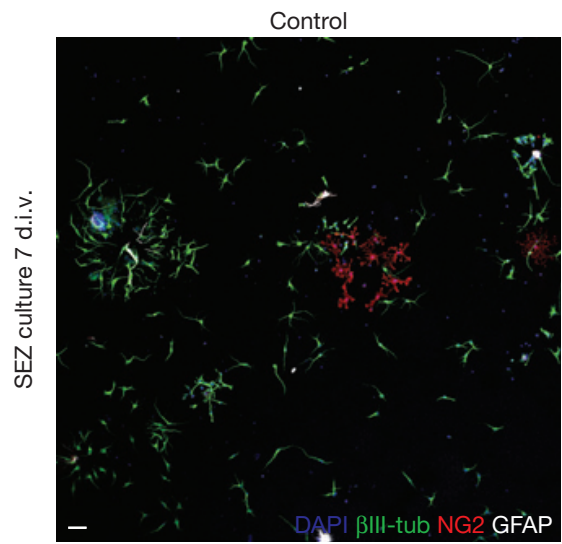

b

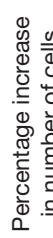

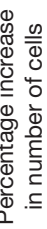

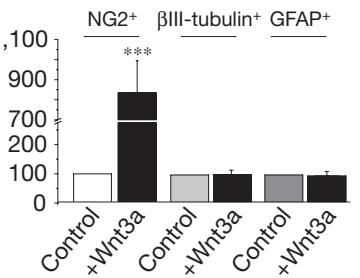

C

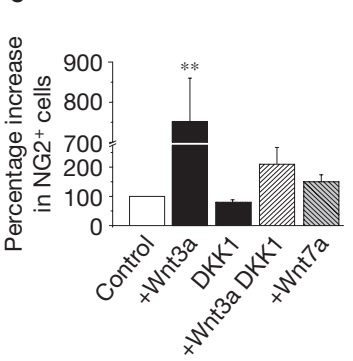

Wnt treatment

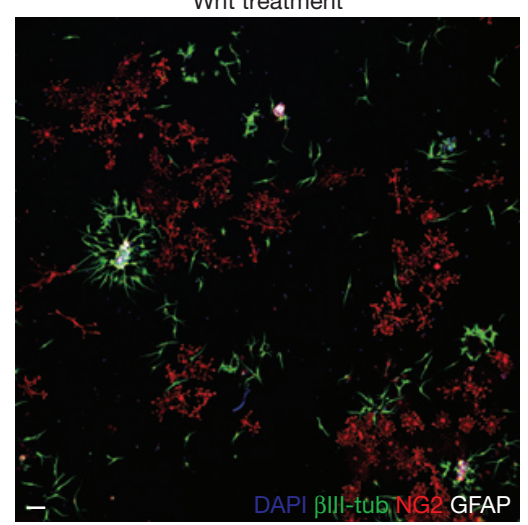

d

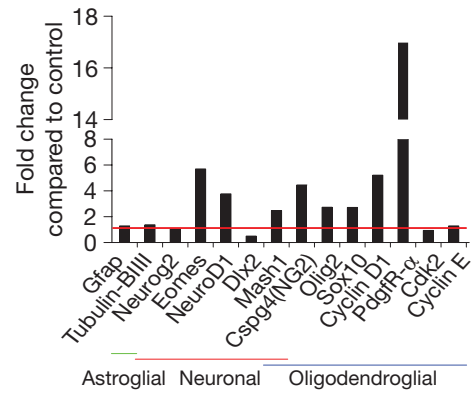

e

SEZ culture 7 d.i.v.
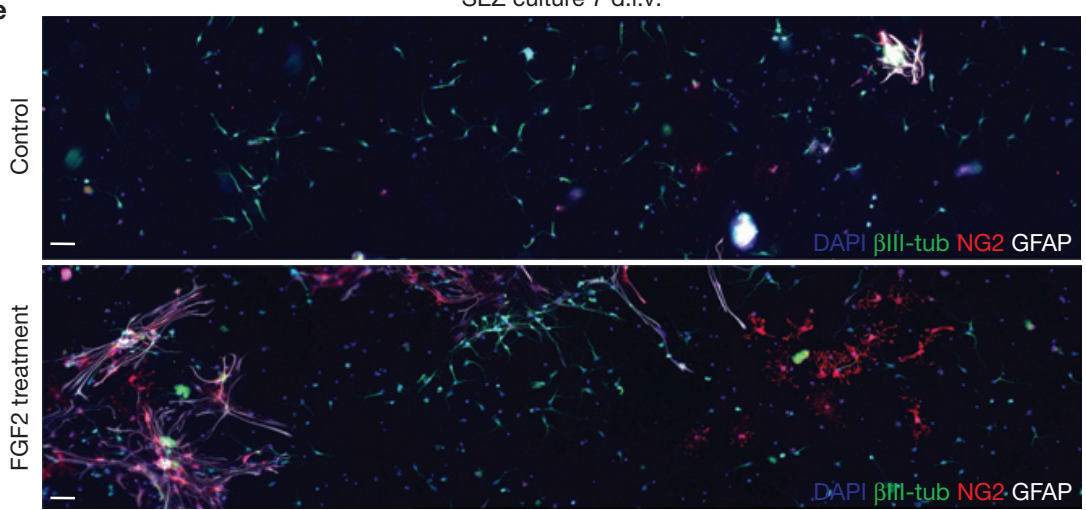

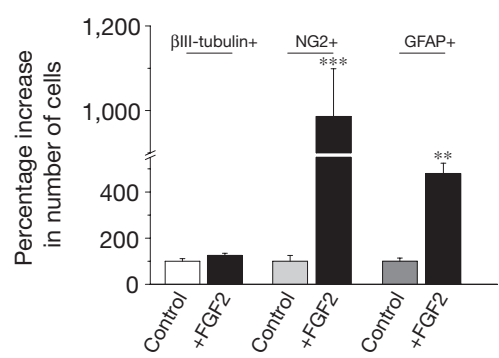

Figure 3 Canonical Wnt signalling augments oligodendrogliogenesis in culture. (a) Control and Wnt3a-treated $\left(200 \mathrm{ng} \mathrm{ml}^{-1}\right)$ cultures. d.i.v., days in vitro. (b) Quantification of the relative increase in NG2-positive oligodendroglia, $\beta$ III-tubulin-positive neurons and GFAP-positive astroglia of Wnt3a-treated cultures when compared with controls (analysis of variance, ANOVA; Tukey's post-test, ${ }^{* * *} P<0.001$, s.e.m. $n=5$ independent experiments). (c) Quantification of the relative increase in oligodendroglia following treatment with Wnt3a, DKK1, Wnt3a plus DKK1, and the non-canonical Wnt agonist Wnt7a (ANOVA, Tukey's post-test, ${ }^{* *}=P<0.01$, s.e.m. $n=3$ independent experiments). (d) Quantitative RT-PCR for mRNAs expressed in the astroglial, neuronal and oligodendroglial lineage in control and Wnt3a-treated cultures. The graph shows means obtained from two independent experiments. (e) FGF2 treatment increases the number of

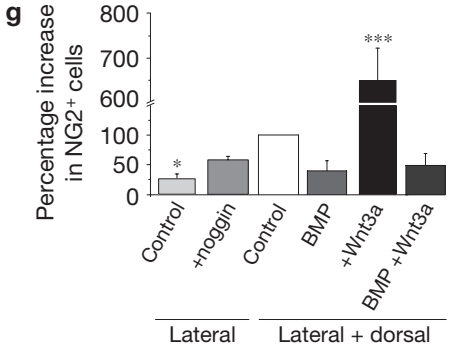

NG2- and GFAP-positive cells in culture. (f) Quantification of the relative increase in $\beta$ III-tubulin-positive neurons, NG2-positive oligodendroglia and GFAP-positive astroglia in FGF2-treated cultures when compared with controls (ANOVA, Tukey's post-test, ${ }^{* *}=P<0.01,{ }^{* * *}=P<0.001$, s.e.m. $n=3$ independent experiments). (g) Effect of BMP signalling on the number of oligodendroglia in cultures of the lateral and lateral + dorsal SEZ. All data were normalized to the control culture of the lateral + dorsal SEZ. Oligodendrogliogenesis was increased in cultures of the lateral SEZ by treatment with the BMP inhibitor noggin $\left(250 \mathrm{ng} \mathrm{ml}^{-1}\right)$. Conversely, BMP4 $\left(1 \mu \mathrm{g} \mathrm{ml}^{-1}\right.$ ) reduced the number of oligodendroglia under control conditions and following Wnt3a treatment (ANOVA, Tukey's post-test, ${ }^{*}=P<0.05$, ${ }^{* * *}=P<0.001$, s.e.m. $n=3$ independent experiments). Scale bars represent $50 \mu \mathrm{m}$. 

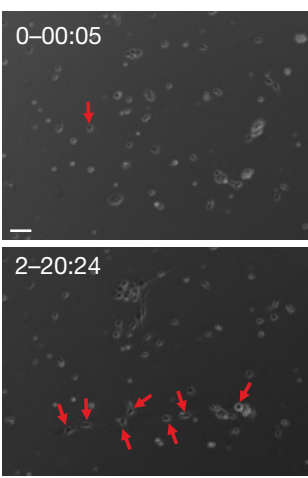

b
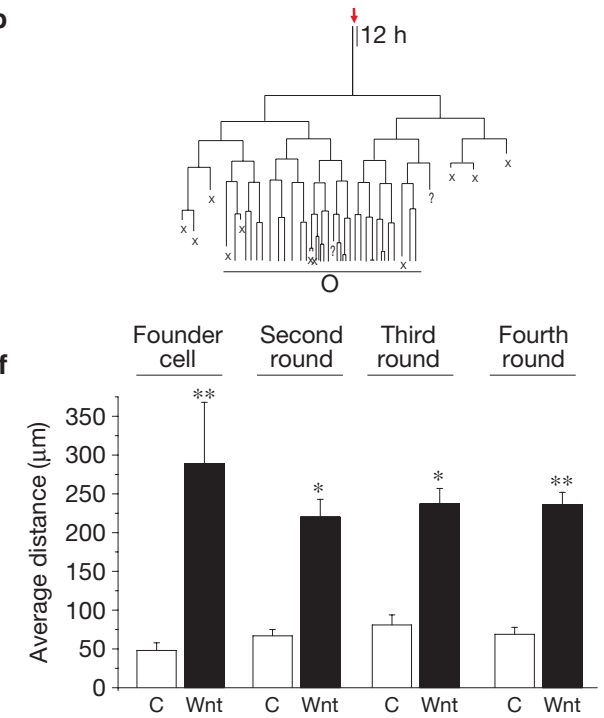
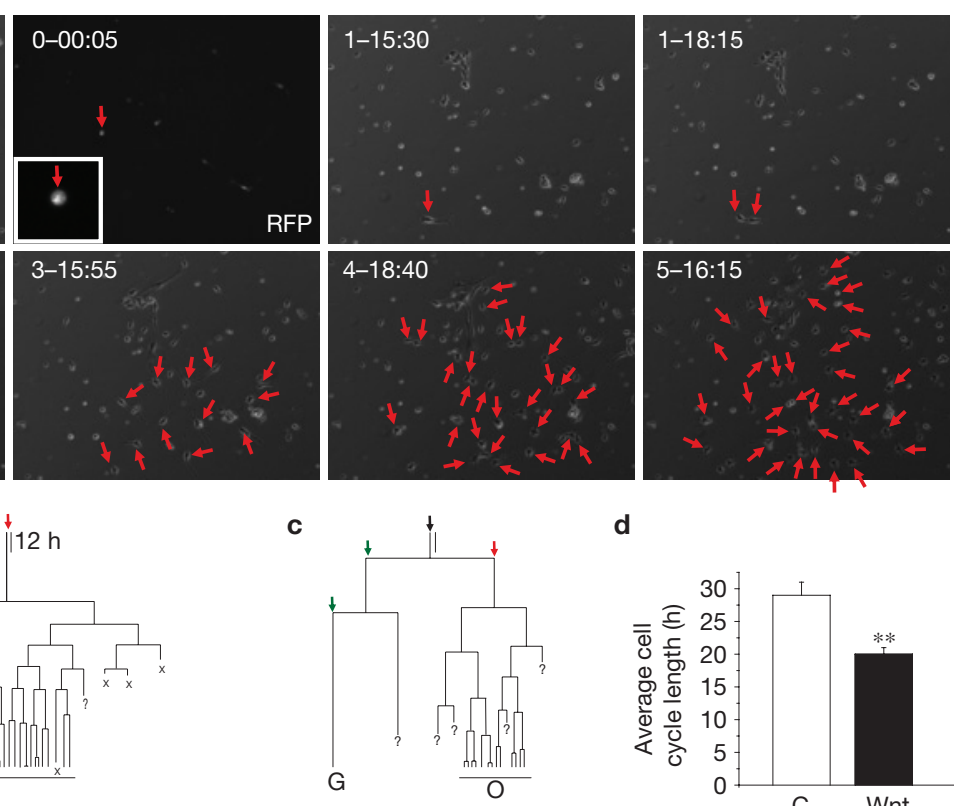

g

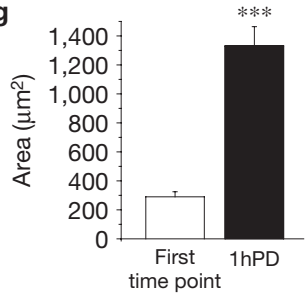

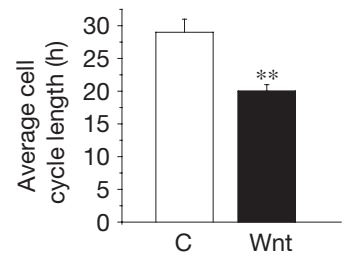
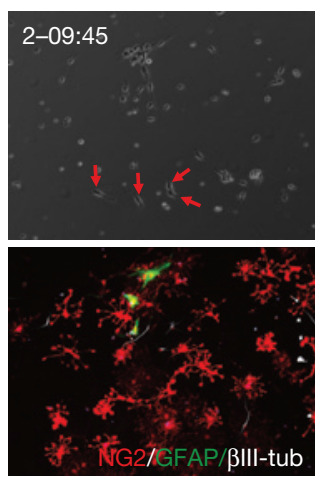

e

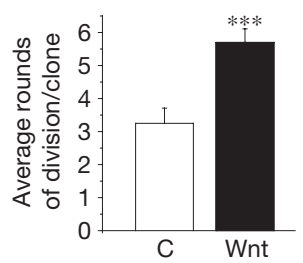

Figure 4 Oligodendroglio- and neurogenic lineage trees tracked by live imaging following Wnt3a treatment. (a) Phase contrast and RFP-fluorescence microscopy images obtained by time-lapse video microscopy of a Wnt3a-treated culture prepared from the adult SEZ of an hGFAP-RFP transgenic mouse; the second image and inset show RFP fluorescence of the clonal founder cell; note the substantial migration of the founder cell between times 0-00:05 and 1-15:30; red arrows point to the progeny at different times; last image post-imaging immunocytochemistry for NG2 (red), GFAP (green) and $\beta \mathrm{III}$-tubulin (white). Scale bar, $50 \mu \mathrm{m}$. (b) Symmetric lineage tree comprising oligodendroglial progeny $(0)$ as tracked from live imaging experiment depicted in a. (c) Example of asymmetric lineage comprising oligodendroglia and astroglia (G). (d) Quantification of the average cell cycle length of all dividing cells tracked within oligodendrogliogenic lineage trees in control (C) and Wnt3a-treated cultures ( $t$-test, ${ }^{* *} P<0.01$, s.e.m. $n=23$ lineage trees obtained by gathering data of three independent experiments; variability represents an aggregation over three experiments).

lineage, because the neuronal and astroglial populations were not affected by local overexpression of Wnt3 (Fig. 7a,b). To corroborate the effect of Wnt signalling on the oligodendroglial lineage, we infused Wnt3a or artificial cerebrospinal fluid over 7 days using osmotic minipumps into the lateral ventricle, followed by 7 days without infusion before killing the animals. As expected, direct infusion markedly increased the number of Olig2-positive cells in the lateral SEZ (99 Olig2-positive cells $0.075 \mathrm{~mm}^{-2}$ in controls versus 589 cells $0.240 \mathrm{~mm}^{-2}$ following Wnt3a infusion ( $n=2$ animals); Fig. 7c). Carrying out these experiments in GLAST :: ${ }^{\text {CreERT2/GFP }}$ mice revealed that part of the effect of Wnt3a treatment comprised oligodendroglia derived from reporter-positive cells, supporting the (e) Average number of rounds of division per oligodendrogliogenic clone in control and Wnt3a-treated cultures (excluding non-dividing cells; $t$-test, ${ }^{* * *} P<0.001$, s.e.m. $n=23$ lineage trees obtained by gathering data of three independent experiments; variability represents an aggregation over three experiments). (f) Quantitative analysis of the distance travelled by the founder cells and subsequent progenies within oligodendroglial clones (verified by post-imaging immunocytochemistry) in control and Wnt3a-treated cultures (ANOVA, Tukey's post-test, ${ }^{*} P<0.05$; ${ }^{* *} P<0.01$, s.e.m. $n=23$ lineage trees obtained by gathering data of three independent experiments; variability represents an aggregation over three experiments). (g) Quantification of the cell size of the founder cells of oligodendrogliogenic clones at the start of the experiment and $1 \mathrm{~h}$ before division (1hPD; $t$-test, ${ }^{* * *} P<0.001$, s.e.m. $n=12$ cells obtained by gathering data of three independent experiments; variability represents an aggregation over three experiments). (h) Example of an asymmetric lineage tree comprising both neurons $(\mathrm{N})$ and astroglia.

notion that canonical Wnt signalling enhances oligodendrogliogenesis from aNSCs (Fig. 7c). Conversely, we carried out injections of a lentivirus encoding for a dominant-negative form of TCF4 (dnTCF4; ref. 32), a downstream target of the canonical Wnt signalling pathway regulator $\beta$-catenin, to inhibit Wnt signalling in the dorsal SEZ. After 14 days, we observed a significant reduction in the percentage of reporter- and Olig2-double-positive cells (from $54 \pm 11 \%$ control to $17 \pm 6 \%$ with dnTCF4) as well as in the reporter- and PDGFR $\alpha$ double-positive cells (from $38 \pm 7 \%$ in controls to $20 \pm 7 \%$ with dnTCF4) (Fig. 6g-i). To identify the source of Wnt3 in the adult SEZ, we carried out fluorescence-activated cell sorting (FACS) analysis to isolate fractions containing specific cell populations following a 
a
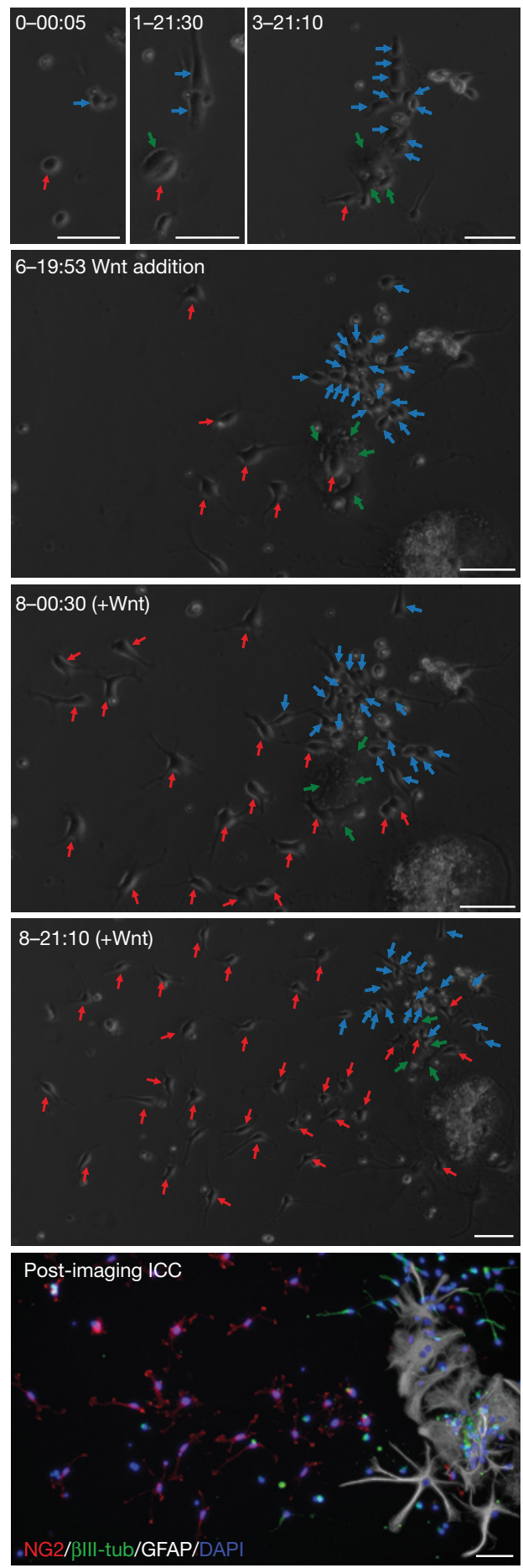

Figure 5 Wnt3a treatment increases the proliferation of the oligodendroglial progenitors without inducing a switch in cell fate. (a) Phase contrast microscopy images obtained by time-lapse video microscopy at different times before and after treatment with Wnt3a; last image, post-imaging immunocytochemistry for NG2 (red), $\beta$ III-tubulin (green) and GFAP (white). Scale bars, $50 \mu \mathrm{m}$. (b) Lineage trees generating neuronal progeny do not respond to subsequent Wnt3a addition (marked by the green line) following a control period. The lineage tree marked by the blue arrow indicates the

protocol previously described ${ }^{33}$. Quantitative PCR following reverse transcription (RT-PCR) revealed that Wnt 3 is expressed mainly by the $\mathrm{O}^{+} / \mathrm{EGFR}^{+}$(OPC) fraction in the adult SEZ (Fig. 6f). b
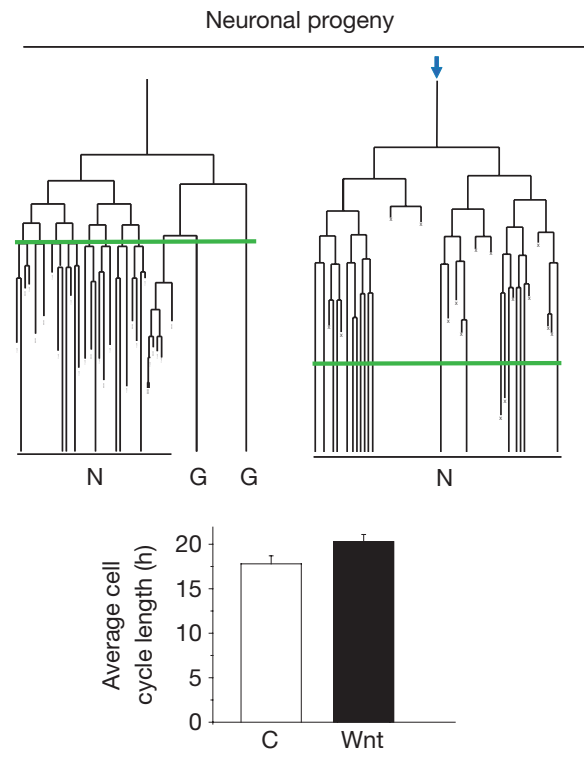

c

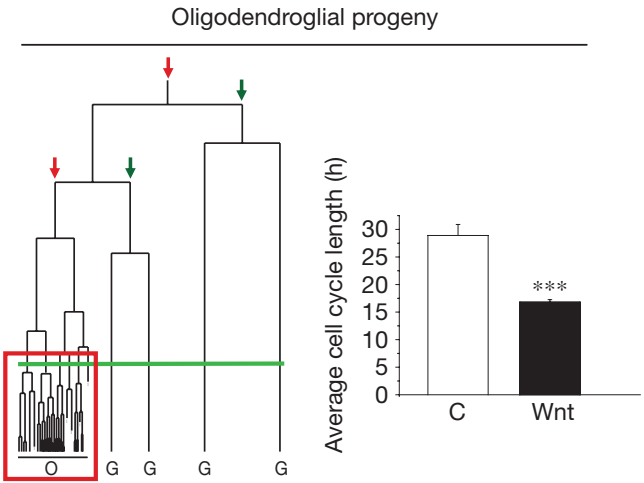

neurogenic clone in a. Note the absence of a change in cell cycle length as shown in the histogram ( $t$-test, s.e.m. $n=7$ independent experiments; C, control). (c) Wnt3a treatment accelerates cell cycle progression in oligodendroglial progenitors. Depicted is the lineage tree shown in a; note the colour code of the arrows marking oligodendroglia, red, and astroglia, green). The histogram indicates the average cell cycle length of all dividing oligodendroglial precursors following Wnt3a treatment ( $t$-test, ${ }^{* * *}=P<0.001$, s.e.m. $n=7$ independent experiments).

Finally, we analysed whether local Wnt3 secretion in the SEZ in vivo also enhances proliferation by counting the number of Ki67-positive cells within the area defined by the expression domain 
a

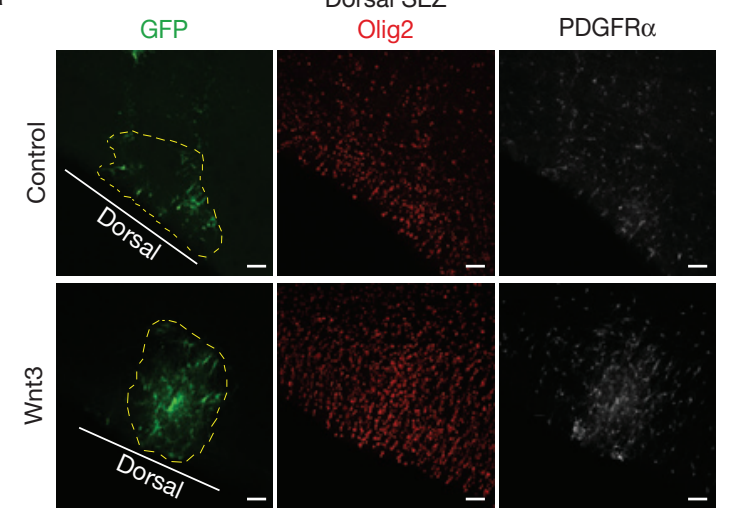

g

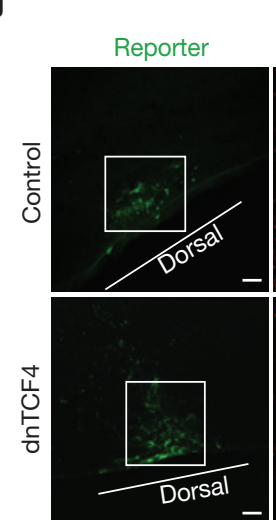

Dorsal SEZ

Olig2

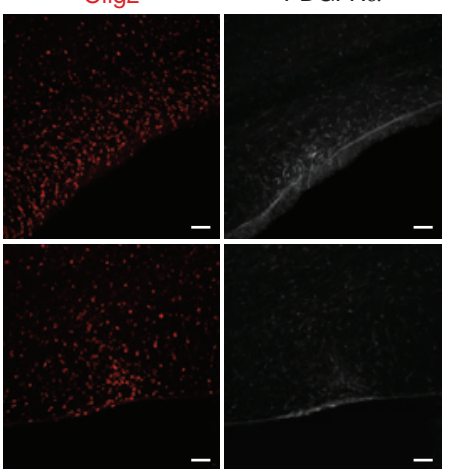

Figure 6 Local overexpression of Wnt3 enhances oligodendrogliogenesis whereas inhibition of canonical Wnt signalling decreases oligodendrogliogenesis in the adult SEZ. (a) Effect of lentivirus-mediated Wnt3 expression in the dorsal wall of the SEZ. Note the increment in Olig2 (red) and PDGFR $\alpha$ (white) expression after Wnt3 overexpression. Olig2- and PDGFR $\alpha$-positive cells were quantified within the marked area. (b) Quantification of the increase in Olig2-positive cells after local Wnt3 expression in the lateral and dorsal SEZ (ANOVA, Tukey's post-test, ${ }^{*} P<0.05$, s.e.m. $n=4$ independent experiments). (c) Quantification of the increase in PDGFR $\alpha$-positive cells after local Wnt3 expression in the lateral and dorsal SEZ (ANOVA, Tukey's post-test, ${ }^{*} P<0.05$, s.e.m. $n=4$ independent experiments). (d) Scheme representing the anatomical site where the lentiviral constructs were sterotactically injected. (e) Comparison of the mRNA levels of dishevelled 1 (Dvl1), LRP-5 co-receptors (Lrp5), Wnt3 and Wnt3a between the dorsal and lateral adult SEZ. Note the higher expression in the dorsal SEZ of Dvl1, Lrp5 and Wnt3, whereas Wnt3a was not detected in either. The graph shows means obtained from two independent

of the Wnt3-encoding lentivirus (Fig. 8). Consistent with the in vitro live imaging data, the number of proliferating cells within the ectopic Wnt3-expression domain incremented to approximately $250 \%(2,796$ Wnt3-treated versus 1,147 control-treated cells $\mathrm{mm}^{-2}$; Fig. 8b). These data support the notion that canonical Wnt signalling exerts its effect on the oligodendroglial lineage through an enhancement of proliferation.

\section{DISCUSSION}

Although the adult SEZ contains stem cells capable of generating neurons and oligodendroglia, single-cell tracking in vitro provides evidence that these two neural lineages have distinct pedigrees. Both arise from aNSCs of astro-/radial glial identity, but in no instance did we observe a clonal founder cell generating simultaneously neuronal

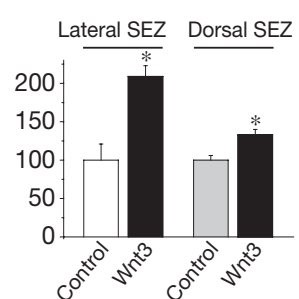

d

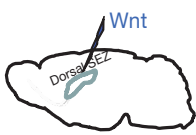

e
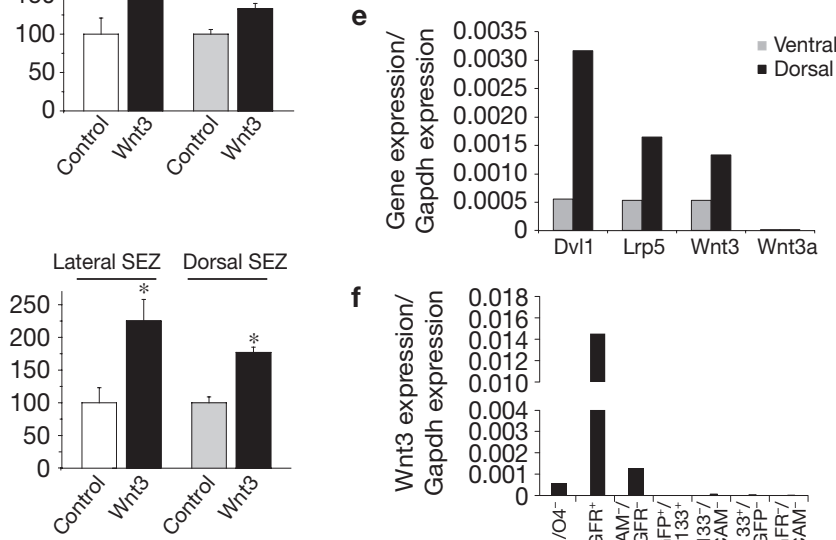

f
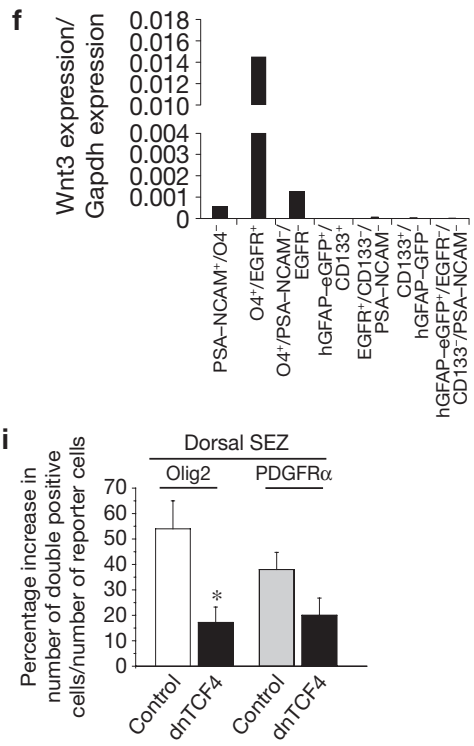

experiments. (f) Expression of Wnt3 among the different cell populations located in the adult SEZ, analysed by quantitative RT-PCR following FACS using the indicated markers (populations enriched in ependymal cells, $\mathrm{CD} 133^{+} /$hGFAP-GFP $^{-}$; aNSCs, hGFAP-eGFP ${ }^{+} / \mathrm{CD} 133^{+}$(eGFP, enhanced

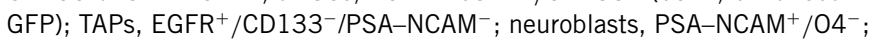

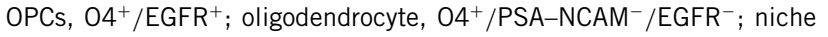
astrocytes, hGFAP-eGFP ${ }^{+} / \mathrm{EGFR}^{-} / \mathrm{CD} 133^{-} / \mathrm{PSA}^{-\mathrm{NCAM}^{-}}{ }^{-}$. The graph shows means obtained from two independent experiments. (g) Effect of lentivirus-mediated expression of dnTCF4, an inhibitor of the canonical Wnt signalling. Note the reduction in Olig2 (red) and PDGFR $\alpha$ (white) positive cells. (h) Higher magnification views of the white boxes depicted in $\mathbf{f}$; note the reduction in the reporter and Olig2 double-positive cells after dnTCF4 expression. (i) Quantification of the reporter and Olig2 or reporter and PDGFR $\alpha$ double-positive cells as a percentage of reporter-positive cells after dnTCF4 expression in the lateral and dorsal SEZ (ANOVA, Tukey's post-test, ${ }^{*} P<0.05$, s.e.m. $n=3$ independent experiments). Scale bars, $50 \mu \mathrm{m}$ $(\mathbf{a}, \mathbf{g}, \mathbf{h})$.

and oligodendroglial offspring. Moreover, canonical Wnt signalling promoted proliferation selectively within the oligodendrogliogenic lineage without affecting the neuronal lineage.

\section{Astro-/radial glial NSCs give rise to oligodendroglial progeny}

Consistent with previous reports ${ }^{4,34}$, we find that the SEZ contains aNSCs generating oligodendroglia. First, we observed that oligodendrogliogenic clones were initiated by cells with an active hGFAP promoter, a defining feature of aNSCs (refs 4,13,18,19). GFAP expression distinguishes oligodendroglia-producing cells of the SEZ from those of other forebrain regions such as the corpus callosum and the cerebral cortex ${ }^{4}$. Furthermore, genetic fate-mapping experiments corroborated the aNSC origin of SEZ oligodendroglia (Fig. 2g). Moreover, 
a
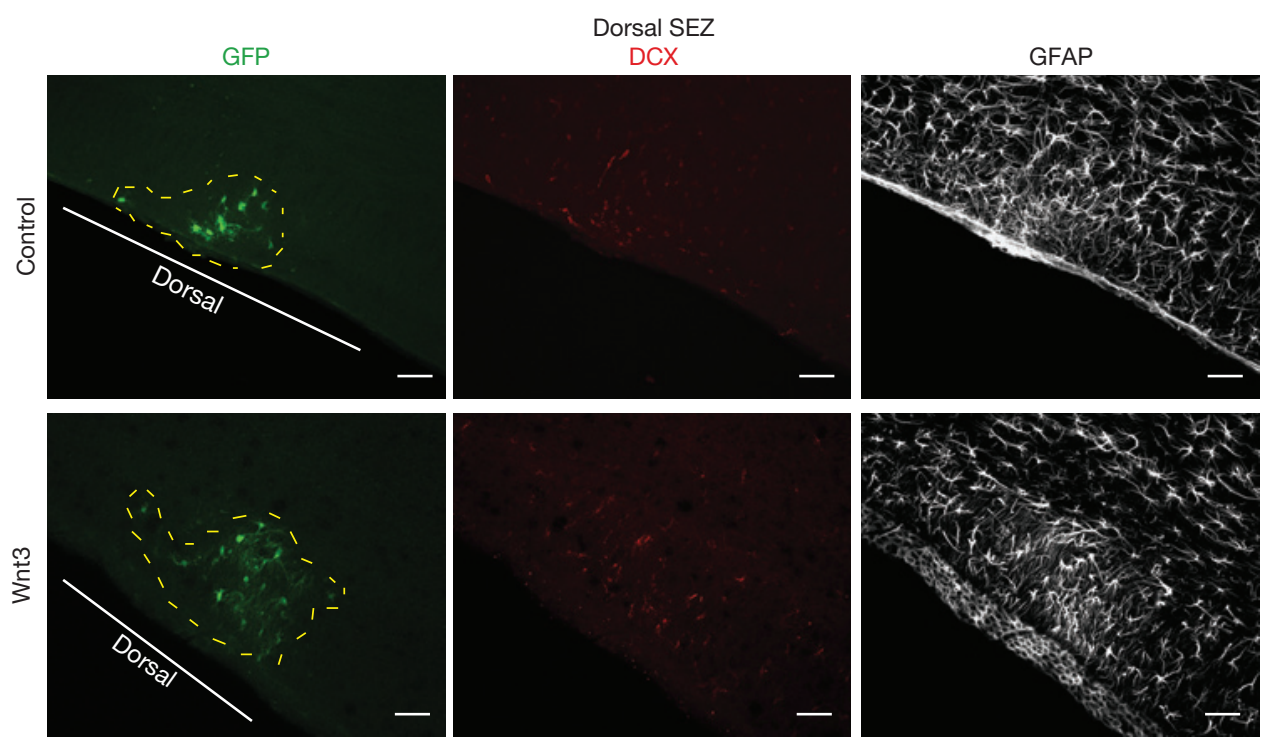

b
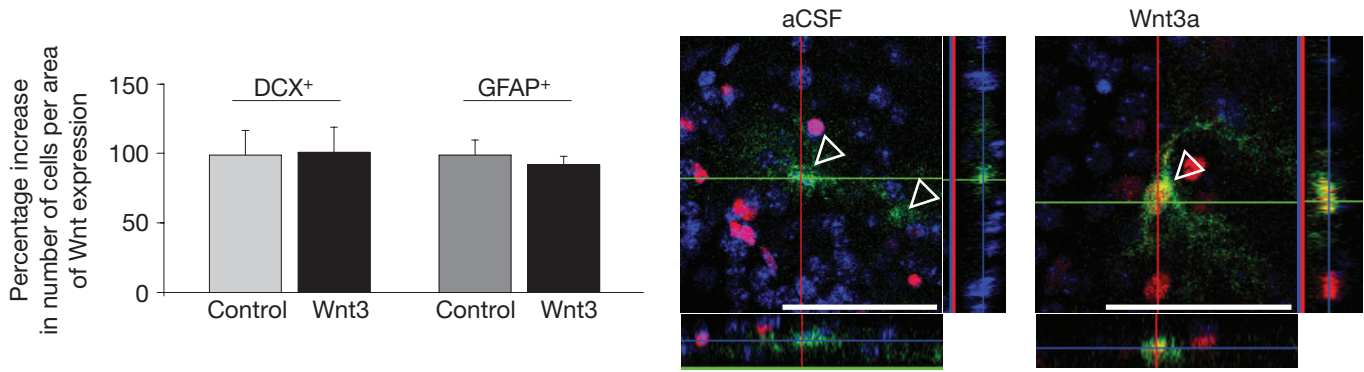

C

GFP

Olig2

Merge

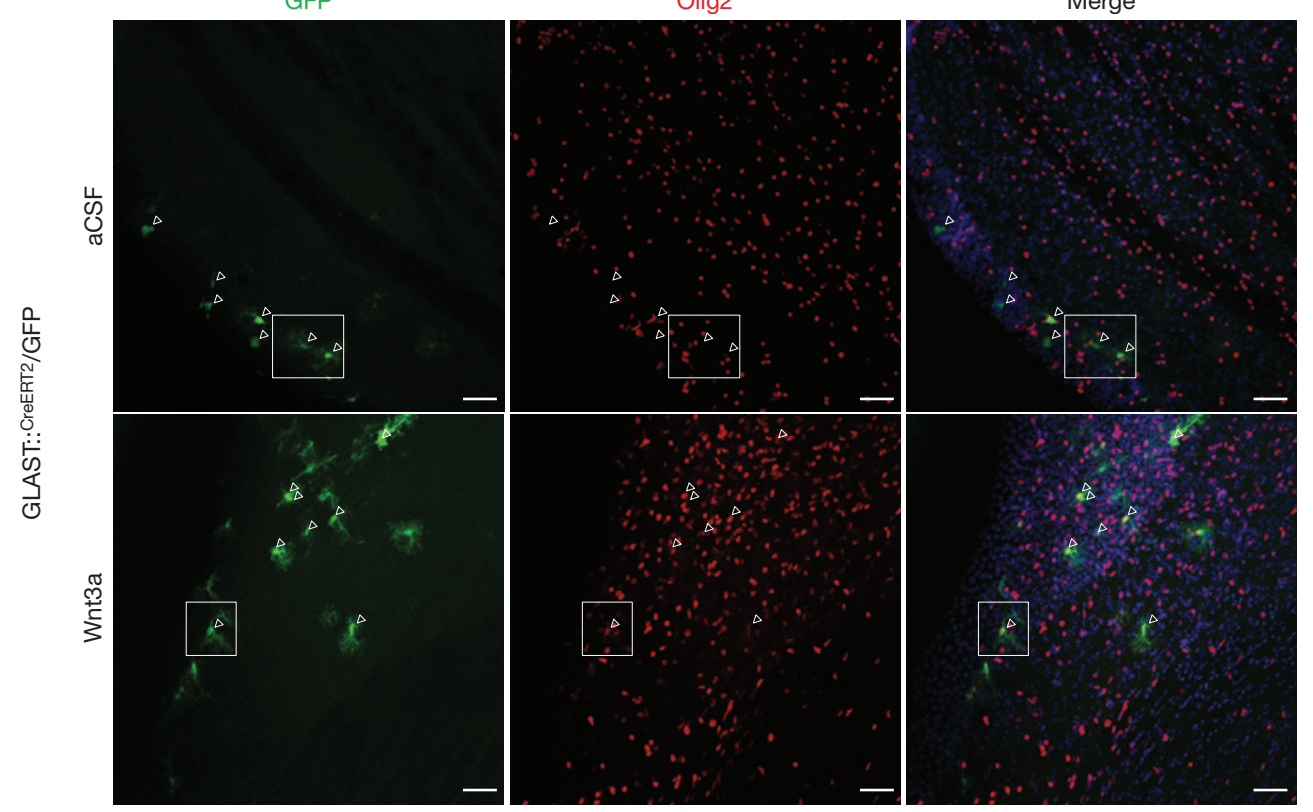

Figure 7 Local Wnt3 overexpression increases oligondendrogliogenesis in the adult SEZ without altering the neuronal and astroglial population. (a) Effect of lentivirus-mediated Wnt3 overexpression on the number of DCX-positive neurons and GFAP-positive astroglia in the dorsal wall of the SEZ. Note that the local overexpression of Wnt3 did not modify the number of neuronal or astroglial populations. DCX- and GFAP-positive cells were quantified within the marked area. (b) Quantification of the increase in DCX- and GFAP-positive cells after local Wnt3 expression in the dorsal SEZ (ANOVA, Tukey's post-test, s.e.m. $n=4$ independent experiments). (c) Chronic infusion of recombinant Wnt3a protein (250 ng per day) into the lateral ventricle of GLAST :: CreERT2/GFP mice results in an increase in the number of Olig2-positive cells when compared with infusion of aCSF as control. Note that several of the Olig2-positive cells originated from reporter-positive cells, indicating their origin from astro-/radial glial aNSCs. Boxed areas are shown above to illustrate the absence (aCSF) or presence (Wnt3a) of Olig2 in fate-mapped progeny in single-stack confocal microscopy images. Scale bars, $50 \mu \mathrm{m}$. 

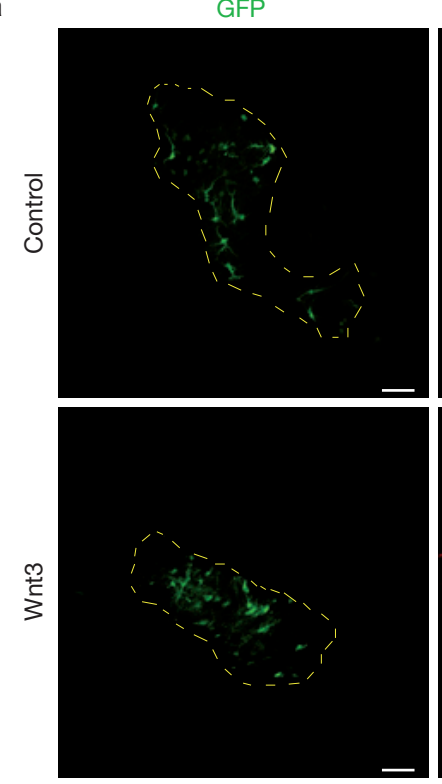

Figure 8 Local Wnt3 overexpression augments proliferation in the adult SEZ. (a) Effect of lentivirus-mediated Wnt3 expression in the dorsal wall of the SEZ. Note the increment in Ki67 (red) after Wnt3 overexpression. Ki67-positive cells were quantified

oligodendrogliogenic lineage trees frequently exhibited asymmetric cell division generating both oligodendroglia and astroglia, in contrast to OPCs of other forebrain regions ${ }^{35}$. Finally, none of the cells characterized by an active GFAP promoter expressed NG2 at the onset. Thus, these data indicate that the oligodendroglial progeny observed in the adult SEZ derives from aNSC rather than pre-differentiated OPCs.

\section{Oligodendrogliogenic lineage progression}

Continuous live imaging of aNSCs and their progeny in vitro revealed that oligodendrogliogenic lineage trees are characterized by amplification of the clone through proliferation of fast-dividing TAPs. Oligodendrogliogenic TAPs are characterized by a high degree of migratory behaviour, whereas neurogenic TAPs typically remain closely associated before reaching the neuroblast stage, when the propensity for migratory behaviour markedly increases ${ }^{16}$. This suggests that siblings within a neurogenic clone are likely to exert stronger influences on one another through cell-to-cell signalling than those of oligodendrogliogenic clones. The differences in migratory properties between these two lineages may reflect their distinct behaviour in vivo $o^{2,4,36-38}$.

\section{The neurogenic and the oligodendrogliogenic progenies constitute distinct lineages}

One of the key findings of this study is the observation that clonal founder cells give rise selectively to neuronal (and astroglial) or oligodendroglial (and astroglial) progenies, but never mixed ones. The analysis of the molecular profile of the clonal founder cells suggested that Pax6 and Olig2 are expressed by neurogenic and oligodendrogliogenic aNSCs, respectively. This strict separation between the neuronal and oligodendroglial lineages observed here seems to be at odds with the fact that during development neurons and oligodendroglia are often generated in a sequential manner within the same progenitor domain ${ }^{39}$, and is even more surprising because

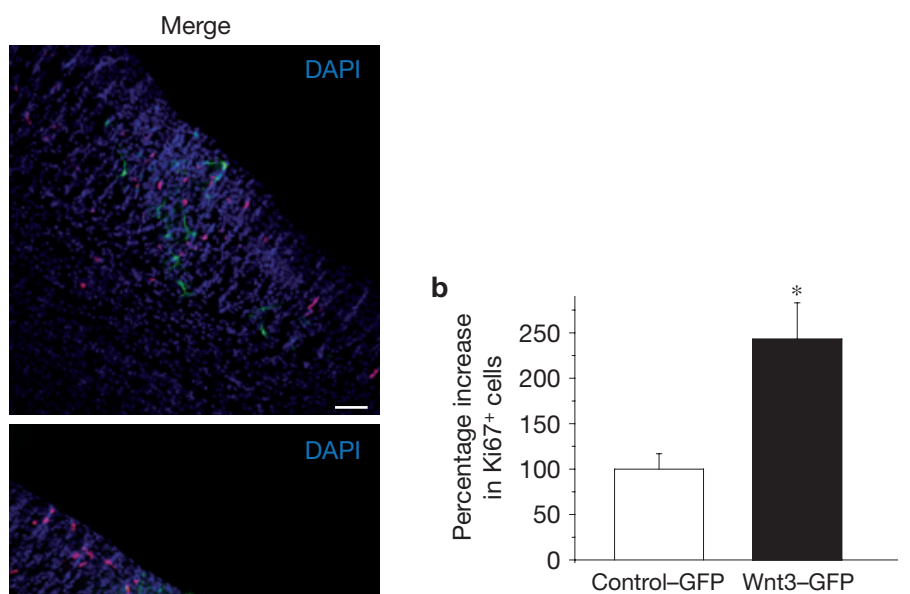

within the marked area. Scale bars, $50 \mu \mathrm{m}$. (b) Quantification of the increase in Ki67-positive cells after local Wnt3 expression in the dorsal SEZ ( $t$-test, ${ }^{*} P<0.05$, s.e.m. $n=4$ independent experiments).

a classical hallmark of aNSCs is their supposed ability to generate neurons, astroglia and oligodendroglia on a clonal level using the neurosphere assay ${ }^{28,40}$. However, this assay requires the stimulation of aNSCs with EGF and FGF2. Studying the clonogenic competence of spinal cord neurospheres, FGF-2 has been shown to confer trilineage differentiation capacity on cells that are only bipotent in $v i v o^{41}$. Likewise, EGF was previously found to induce massive astro- ${ }^{16,28}$ and oligodendrogliogenesis ${ }^{34,42}$ from SEZ aNSCs.

The fact that the dorsal SEZ harbours more oligodendrogliogenic aNSCs than the lateral SEZ is consistent with the notion of a mosaic organization of the SEZ (ref. 11). According to this notion, different germinative domains within the SEZ are of distinct embryonic origins along the dorsoventral axis of the forebrain ${ }^{12}$, which in turn accounts for their respective competence in generating distinct progeny. Future studies are required to define the regional and temporal origins of the various neuro- and oligodendrogliogenic aNSCs in the adult SEZ during embryogenesis.

\section{Canonical Wnt signalling selectively stimulates proliferation within the oligodendrogliogenic lineage}

Moreover, apart from distinct embryonic origins the fate choice of aNSCs can also be affected by local signalling. Indeed, it has been previously shown that sonic hedgehog signalling determines aNSC positional identity ${ }^{9}$. Here we observed that stimulation with Wnt3/3a results in a massive expansion of oligodendrogliogenic clones without affecting the generation of other cell types both in vitro and in vivo. Canonical Wnt signalling has been reported to be particularly high in the dorsal SEZ (ref. 20). Accordingly, we observed that mRNAs encoding for several components of the Wnt canonical pathway are expressed at higher levels in the dorsal SEZ when compared with the lateral SEZ. These data, together with the fact that gain- or loss-of-function of Wnt signalling increases or decreases oligodendrogliogenesis, respectively, 
suggest that local Wnt signalling contributes to the enrichment of oligodendroglia in the dorsal SEZ. Conversely, local BMP signalling has been shown to repress an oligodendrogliogenic fate in the lateral SEZ (ref. 14). Thus, largely non-overlapping domains of high Wnt and BMP signalling seem to define the level of oligodendrogliogenesis in the adult SEZ. Of note, mRNA expression analysis suggests that Wnt3 is the physiologically relevant player, whereas Wnt3a was hardly detectable within adult SEZ tissue. Intriguingly, FACS analysis suggests that the cell type expressing the highest amount of Wnt 3 mRNA comprises cells of the oligodendroglial lineage. This suggests that, in the adult SEZ, regions of higher density of Wnt3-secreting oligodendroglial precursor cells promote local enhancement of oligodendrogliogenesis through a positive feedback loop.

Whereas canonical Wnt signalling has been implicated in the regulation of adult neurogenesis in the dentate gyrus ${ }^{31}$, we did not observe an obvious effect of Wnt3/3a treatment on SEZ neurogenesis. These data provide support for the notion of the separation of the neuroand oligodendrogliogenic lineages in vivo and may represent distinct cellular targets of intervention in therapeutic contexts. However, Wnt3a treatment of SEZ cultures augmented the expression of fate determinants such as Eomes (tbr2) and neurod1. This indicates that canonical Wnt signalling regulates the differentiation rather than the expansion of neurons belonging to the glutamatergic lineage $e^{43}$, also enriched in the dorsal SEZ (ref. 10).

To our knowledge, the present study is the first to report a massive effect of canonical Wnt signalling on the proliferation within the oligodendroglial lineage, although previous studies have shown that Wnt signalling maintains OPCs in an immature state ${ }^{4-46}$. Moreover, after traumatic brain injury, the canonical Wnt signal transducer $\beta$-catenin translocates to the nucleus of proliferating NG2 progenitor cells in the cerebral cortex ${ }^{47}$, potentially contributing to the increase in NG2 progenitor cells after stab wound injury ${ }^{48}$.

Several lines of evidence suggest that the effect of Wnt signalling on oligodendrogenesis is primarily due to a selective activation of proliferation within the oligodendrogliogenic lineage and less likely to be due to a switch in cell fate. First, whereas Wnt3a stimulated massive expansion of NG2-positive oligodendroglia, no concomitant decrease in neurogenesis was observed. Second, Wnt3a treatment caused a marked reduction in cell cycle length and simultaneous increase in cell divisions selectively within the oligodendrogliogenic lineage. Third, Wnt treatment did not cause the appearance of mixed clones (containing both neurons and oligodendroglia), a finding incompatible with canonical Wnt signalling acting by diverting neurogenic TAPs towards oligodendrogliogenesis.

Thus, in addition to previously described restrictions regarding neuronal subtype specification ${ }^{11}$, our study reveals that aNSCs in the adult SEZ are also lineage restricted with respect to the decision of acquiring a neuronal or an oligodendroglial identity, and that these lineages are differentially regulated by regionalized niche signals such as Wnts.

\section{METHODS}

Methods and any associated references are available in the online version of the paper.

Note: Supplementary Information is available in the online version of the paper

\section{ACKNOWLEDGEMENTS}

We are grateful to M. Götz (LMU Munich) for discussion and valuable comments on the manuscript. We also thank T. Simon-Ebert and G. Jaeger for excellent technical assistance. We are indebted to the Graduate School of Systemic Neuroscience for financial support of the time-lapse video microscope. This work was supported by grants of the Bundesministerium für Bildung und Forschung (01GN 1009A), the Bavarian State Ministry of Sciences, Research and the Arts (ForNeuroCell), and the Belgian Science Policy Office (P7/20 Wibrain) to B.B. and the Deutsche Forschungsgemeinschaft to B.B. (BE 4182/2-2) and T.S. (SCHR 1142/1-2).

\section{AUTHOR CONTRIBUTIONS}

Experiments were designed by F.O. and B.B. F.O. carried out most of the experiments, analysed the data and prepared the figures. S.G. demonstrated Wnt3 secretion from overexpressing cells. G.M. carried out and analysed RT-PCR experiments. A.D., C.S. and L.D. helped with the in vivo experiments. J.F. carried out the FACS analysis. D.C.L. contributed the Wnt3 lentivirus. T.S. helped with the time-lapse experiments and contributed the analysis software. F.O and B.B. wrote the manuscript.

\section{COMPETING FINANCIAL INTERESTS}

The authors declare no competing financial interests.

Published online at www.nature.com/doifinder/10.1038/ncb2736

Reprints and permissions information is available online at www.nature.com/reprints

1. Ihrie, R. A. \& Alvarez-Buylla, A. Lake-front property: A unique germinal niche by the lateral ventricles of the adult brain. Neuron 70, 674-686 (2011).

2. Lois, C., Garcia-Verdugo, J. M. \& Alvarez-Buylla, A. Chain migration of neuronal precursors. Science 271, 978-981 (1996).

3. Gonzalez-Perez, O. \& Alvarez-Buylla, A. Oligodendrogenesis in the subventricular zone and the role of epidermal growth factor. Brain Res. Rev. 67, 147-156 (2011).

4. Menn, B. et al. Origin of oligodendrocytes in the subventricular zone of the adult brain. J. Neurosci. 26, 7907-7918 (2006).

5. Hack, M. A. et al. Neuronal fate determinants of adult olfactory bulb neurogenesis. Nat. Neurosci. 8, 865-872 (2005).

6. Picard-Riera, N. et al. Experimental autoimmune encephalomyelitis mobilizes neural progenitors from the subventricular zone to undergo oligodendrogenesis in adult mice. Proc. Natl Acad. Sci. USA 99, 13211-13216 (2002).

7. Doetsch, F., Caille, I., Lim, D. A., Garcia-Verdugo, J. M. \& Alvarez-Buylla, A. Subventricular zone astrocytes are neural stem cells in the adult mammalian brain. Cell 97, 703-716 (1999).

8. Mirzadeh, Z., Merkle, F. T., Soriano-Navarro, M., Garcia-Verdugo, J. M. \& Alvarez-Buylla, A. Neural stem cells confer unique pinwheel architecture to the ventricular surface in neurogenic regions of the adult brain. Cell Stem Cell 3, 265-278 (2008).

9. Ihrie, R. A. et al. Persistent sonic hedgehog signalling in adult brain determines neural stem cell positional identity. Neuron 71, 250-262 (2011).

10. Brill, M. S. et al. Adult generation of glutamatergic olfactory bulb interneurons. Nat. Neurosci. 12, 1524-1533 (2009).

11. Merkle, F. T., Mirzadeh, Z. \& Alvarez-Buylla, A. Mosaic organization of neural stem cells in the adult brain. Science 317, 381-384 (2007).

12. Kriegstein, A. \& Alvarez-Buylla, A. The glial nature of embryonic and adult neural stem cells. Ann. Rev. Neurosci. 32, 149-184 (2009).

13. Beckervordersandforth, R. et al. In vivo fate mapping and expression analysis reveals molecular hallmarks of prospectively isolated adult neural stem cells. Cell Stem Cell 7, 744-758 (2010).

14. Colak, D. et al. Adult neurogenesis requires Smad4-mediated bone morphogenic protein signalling in stem cells. J. Neurosci. 28, 434-446 (2008).

15. Jablonska, B. et al. Chordin-induced lineage plasticity of adult SVZ neuroblasts after demyelination. Nat. Neurosci. 13, 541-550 (2010).

16. Costa, M. R. et al. Continuous live imaging of adult neural stem cell division and lineage progression in vitro. Development 138, 1057-1068 (2011).

17. Ortega, F. et al. Using an adherent cell culture of the mouse subependymal zone to study the behaviour of adult neural stem cells on a single-cell level. Nat. Protoc. 6, 1847-1859 (2011).

18. Doetsch, F. The glial identity of neural stem cells. Nat. Neurosci. 6, 1127-1134 (2003).

19. Pastrana, E., Cheng, L. C. \& Doetsch, F. Simultaneous prospective purification of adult subventricular zone neural stem cells and their progeny. Proc. Natl Acad. Sci. USA 106, 6387-6392 (2009).

20. Marinaro, C. et al. Wnt signalling has opposing roles in the developing and the adult brain that are modulated by Hipk1. Cereb. Cortex 10, 2415-2427 (2012).

21. Nishiyama, A., Watanabe, M., Yang, Z. \& Bu, J. Identity, distribution, and development of polydendrocytes: NG2-expressing glial cells. J. Neurocytol. 31, 437-455 (2002). 
22. Pringle, N. P., Mudhar, H. S., Collarini, E. J. \& Richardson, W. D. PDGF receptors in the rat CNS: During late neurogenesis, PDGF $\alpha$-receptor expression appears to be restricted to glial cells of the oligodendrocyte lineage. Development $\mathbf{1 1 5}$, 535-551 (1992).

23. Simon, C., Lickert, H., Götz, M. \& Dimou, L. Sox10-iCreER(T2): A mouse line to inducibly trace the neural crest and oligodendrocyte lineage. Genesis 6, 506-515 (2012)

24. Nakamura, T., Colbert, M. C. \& Robbins, J. Neural crest cells retain multipotential characteristics in the developing valves and label the cardiac conduction system. Circ. Res. 98, 1547-1554 (2006).

25. Hirrlinger, P. G. et al. Expression of reef coral fluorescent proteins in the central nervous system of transgenic mice. Mol. Cell Neurosci. 30, 291-303 (2005).

26. Mori, T. et al. Inducible gene deletion in astroglia and radial glia-a valuable tool for functional and lineage analysis. Glia 54, 21-34 (2006).

27. Michaelidis, T. M. \& Lie, D. C. Wnt signalling and neural stem cells: Caught in the Wnt web. Cell Tissue Res. 331, 193-210 (2008).

28. Doetsch, F., Petreanu, L., Caille, I., Garcia-Verdugo, J. M. \& Alvarez-Buylla, A. EGF converts transit-amplifying neurogenic precursors in the adult brain into multipotent stem cells. Neuron 36, 1021-1034 (2002).

29. Matsumoto, Y. et al. Differential proliferation rhythm of neural progenitor and oligodendrocyte precursor cells in the young adult hippocampus. PLoS One 6 e27628 (2011).

30. Semenov, M. V. et al. Head inducer Dickkopf-1 is a ligand for Wnt coreceptor LRP6 Curr. Biol. 11, 951-961 (2001).

31. Lie, D. C. et al. Wnt signalling regulates adult hippocampal neurogenesis. Nature 437, 1370-1375 (2005).

32. Fuerer, C. \& Nusse, R. Lentiviral vectors to probe and manipulate the Wnt signalling pathway. PLoS One 5, e9370 (2010).

33. Fischer, J. et al. Prospective isolation of adult neural stem cells from the mouse subependymal zone. Nat. Protoc. 6, 1981-1989 (2011).

34. Gonzalez-Perez, O., Romero-Rodriguez, R., Soriano-Navarro, M., Garcia-Verdugo, J. M. \& Alvarez-Buylla, A. Epidermal growth factor induces the progeny of subventricular zone type B cells to migrate and differentiate into oligodendrocytes. Stem Cells 27, 2032-2043 (2009).

35. Dimou, L., Simon, C., Kirchhoff, F., Takebayashi, H. \& Götz, M. Progeny of Olig2-expressing progenitors in the grey and white matter of the adult mouse cerebral cortex. J. Neurosci. 28, 10434-10442 (2008).
36. Lois, C. \& Alvarez-Buylla, A. Long-distance neuronal migration in the adult mammalian brain. Science 264, 1145-1148 (1994).

37. Saghatelyan, A. Role of blood vessels in the neuronal migration. Semin. Cell Dev. Biol. 20, 744-750 (2009).

38. Snapyan, M. et al. Vasculature guides migrating neuronal precursors in the adult mammalian forebrain via brain-derived neurotrophic factor signalling. J. Neurosci. 29, 4172-4188 (2009).

39. Li, H., de Faria, J. P., Andrew, P., Nitarska, J. \& Richardson, W. D. Phosphorylation regulates OLIG2 cofactor choice and the motor neuron-oligodendrocyte fate switch. Neuron 69, 918-929 (2011).

40. Chojnacki, A. \& Weiss, S. Production of neurons, astrocytes and oligodendrocytes from mammalian CNS stem cells. Nat. Protoc. 3, 935-940 (2008).

41. Gabay, L., Lowell, S., Rubin, L. L. \& Anderson, D. J. Deregulation of dorsoventral patterning by FGF confers trilineage differentiation capacity on CNS stem cells in vitro. Neuron 40, 485-499 (2003).

42. Aguirre, A. \& Gallo, V. Reduced EGFR signalling in progenitor cells of the adult subventricular zone attenuates oligodendrogenesis after demyelination. Neuron Glia Biol. 3, 209-220 (2007).

43. Hevner, R. F., Hodge, R. D., Daza, R. A. \& Englund, C. Transcription factors in glutamatergic neurogenesis: conserved programs in neocortex, cerebellum, and adult hippocampus. Neurosci. Res. 55, 223-233 (2006).

44. Chew, L. J. et al. SRY-box containing gene 17 regulates the $\mathrm{Wnt} / \beta$-catenin signalling pathway in oligodendrocyte progenitor cells. J. Neurosci. 31, 13921-13935 (2011).

45. Langseth, A. J. et al. Wnts influence the timing and efficiency of oligodendrocyte precursor cell generation in the telencephalon. J. Neurosci. 30, 13367-13372 (2010).

46. Feigenson, K., Reid, M., See, J., Crenshaw, E. B. 3rd \& Grinspan, J. B. Wnt signalling is sufficient to perturb oligodendrocyte maturation. Mol. Cell Neurosci. 42, 255-265 (2009)

47. White, B. D. et al. $\beta$-catenin signalling increases in proliferating NG2+ progenitors and astrocytes during post-traumatic gliogenesis in the adult brain. Stem Cells 28, 297-307 (2010).

48. Simon, C., Götz, M. \& Dimou, L. Progenitors in the adult cerebral cortex: cell cycle properties and regulation by physiological stimuli and injury. Glia 59, 869-881 (2011). 


\section{METHODS}

Animals. All animal procedures were carried out in accordance with the Policies on the Use of Animals approved by the institute and the state of Upper Bavaria. For wild-type analysis, we used C57BL/6 mice. In addition, we used GLAST :: ${ }^{\text {CreERT2 }}$, hGFAP-RFP (ref. 25), Sox10-iCreERT2 (ref. 23) and CAG CAT GFP reporter ${ }^{24}$ lines. For induction of Cre-mediated recombination, mice received tamoxifen orally (10 mg; dissolved at $40 \mathrm{mg} \mathrm{ml}^{-1}$ in a 1:9 ethanol/corn oil mixture) three times (once a day every second day for $5 \mathrm{~d}$ ). Mice (comprising indistinctly females and males) were adults $2-3$ months of age.

Cell culture. SEZ cultures were prepared from the lateral and dorsal walls of the adult SEZ of young adult (8-12 weeks) C57/Bl6, hGFAP-RFP or

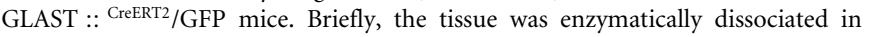
$0.7 \mathrm{mg} \mathrm{ml}^{-1}$ hyaluronic acid (Sigma-Aldrich) and $1.33 \mathrm{mg} \mathrm{ml}^{-1}$ trypsin (SigmaAldrich) in Hanks' balanced salt solution (HBSS; Invitrogen) with $2 \mathrm{mM}$ glucose at $37^{\circ} \mathrm{C}$ for $30 \mathrm{~min}$. Dissociation was stopped by adding an equal volume of an ice-cold medium consisting of $4 \%$ bovine serum albumin (BSA; Sigma-Aldrich) in Earle's balanced salt solution (EBSS; Invitrogen), buffered with 20 mM HEPES (Invitrogen). Cells were then centrifuged at $200 \mathrm{~g}$ for $5 \mathrm{~min}$, resuspended in ice cold medium consisting of $0.9 \mathrm{M}$ sucrose (Sigma-Aldrich) in $0.5 \times$ HBSS, and centrifuged for $10 \mathrm{~min}$ at $750 \mathrm{~g}$. The cell pellet was resuspended in $2 \mathrm{ml}$ ice cold medium consisting of $4 \%$ BSA in EBSS buffered with 2 mM HEPES, and the cell suspension was placed on top of $12 \mathrm{ml}$ of the same medium and centrifuged for $7 \mathrm{~min}$ at $200 \mathrm{~g}$. The cell pellet was finally resuspended in DMEM/F12 Glutamax (Invitrogen) supplemented with B27 (Invitrogen), $2 \mathrm{mM}$ glutamine (Sigma), 100 units $\mathrm{ml}^{-1}$ penicillin (Invitrogen) and $100 \mu \mathrm{g} \mathrm{ml}^{-1}$ streptomycin (Invitrogen) and buffered with $8 \mathrm{mM}$ HEPES, and cells were plated on poly-D-lysine (Sigma) coated coverslips at a density of 200-300 cells $\mathrm{mm}^{-2}$. Cells in culture were treated the first and the second day with only recombinant mouse Wnt3a, $200 \mathrm{ng} \mathrm{m}^{-1}$, Wnt7a (R\&D Systems) or Wnt3a + DKK1 (R\&D Systems).

Immunocytochemistry. Cultures were fixed in 4\% paraformaldehyde in PBS for $15 \mathrm{~min}$ at room temperature and processed for antibody staining. Antibodies used were mouse IgG2b anti- $\beta$ III-tubulin (Sigma-Aldrich; 1:1,000, catalogue no T8660, clone SDL-3D10), mouse IgG1 (m)-GFAP (Sigma; 1:500, catalogue no G3893, clone G-A-5), chicken anti-GFP (Aves Labs; 1:1,000, catalogue no GFP1020), rabbit Olig2 (Millipore; 1:500, catalogue no AB9610), rabbit-NG2 (Millipore; 1:500, catalogue no AB5320), rabbit Pax6 (Millipore; 1:500, catalogue no AB5409) and rat IgG2A Mash1 (R\&D Systems; 1:200, catalogue no MAB2567, clone 290622). Secondary antibodies were chosen according to the primary antibodies and were coupled to Alexa Fluor 488 or FITC (fluorescein isothiocyanate), Cy3, Alexa Fluor 647, Cy5 or biotinylated secondary antibodies, and coverslips were counterstained with 4,6-diamidino-2-phenylindole (DAPI; Sigma Aldrich).

Time-lapse video microscopy. Time-lapse video microscopy and single-cell tracking ${ }^{49-51}$ of primary SEZ cultures was carried out with a cell observer (Zeiss) at a constant temperature of $37^{\circ} \mathrm{C}$ and $8 \% \mathrm{CO}_{2}$. Phase contrast microscopy images were acquired every $5 \mathrm{~min}$ for $6-10$ days using a $\times 20$ phase contrast microscopy objective (Zeiss), an AxioCamHRm camera and Zeiss AxioVision 4.7 software. Single-cell tracking was carried out using a self-written computer program (TTT; ref. 52). Videos were assembled using ImageJ $1.42 \mathrm{q}$ (National Institutes of Health) software and are played at a speed of 1 or 2 frames per second.

FACS. Adult SEZs from hGFAP-eGFP mice were dissected and dissociated as described previously ${ }^{33}$. Cells were stained for $30 \mathrm{~min}$ using fluorochrome labelled antibodies (CD133-PE, 1:250, catalogue no 12-1331, eBioscience (clone 13A4); PSA-NCAM-PE, 1:300, catalogue no 130-093-274, Miltenyi (2-2B; isotype mouse IgM); O4-APC, 1:200, catalogue no 130-095-891, Miltenyi (clone O4; isotype mouse $\operatorname{IgM}$ ) ) and fluorescent ligand of EGF receptor 1:300 (EGF-Alexa Fluor 647, catalogue no E-35351, and EGF-Alexa Fluor 488, catalogue no E-13345, Molecular Probes). Cells were analysed and sorted with a FACS Aria III (BD) using a $100 \mu \mathrm{m}$ nozzle at 20 p.s.i. Debris and aggregated cells were gated out by forward and sideward scatter. Gates were set with isotype controls and wild-type mice. Cells were directly sorted into RLT lysis buffer to isolate RNA using the Qiagen RNeasy Mini Kit.

RNA extraction and real-time PCR. Four wells from untreated and Wnt3atreated SEZ cultured cells were lysed in RLT Plus buffer and total RNA was extracted with an RNeasy Plus Micro Kit (Qiagen), according to the manufacturer's instructions. RNA was retro-transcribed using Super-ScriptIII reverse transcriptase (Invitrogen) and random primers (Roche). Each complementary DNA was diluted one to five, and $1 \mu \mathrm{l}$ was used for each real-time reaction. Real-time PCR was carried out on a LightCycler 480 (Roche) using the LightCycler Probes Master kit (Roche) and Monocolor Hydrolysis Probe/UPL Probe (Roche) according to the manufacturer's instructions ( $20 \mu \mathrm{l}$ final volume). The expression of each gene was analysed in triplicate. Data analysis was carried out with the $\Delta \Delta \mathrm{Ct} \operatorname{method}^{53}$ Quantification was carried out on two independent experiments. Primers are listed in Supplementary Table S1.

Immunohistochemistry. For immunohistochemistry, mice were deeply anaesthetized and killed by transcardial perfusion with PBS and then $4 \%$ paraformaldehyde (wt/vol.) in PBS. Brains were dissected and postfixed overnight in paraformaldehyde at $4{ }^{\circ} \mathrm{C}$. For free-floating vibratome sections, brains were embedded in $4 \%$ agarose solution and sections were cut at a thickness of $70 \mu \mathrm{m}$. Primary antibodies were diluted in $0.1 \mathrm{M}$ PBS containing $0.5 \%$ Triton X-100 (wt/vol.) and 2\% BSA (wt/vol.). Antibodies used were guinea pig anti-DCX (Millipore; 1:500, catalogue no AB2253), chicken anti-GFP (Aves Labs; 1:500), rabbit anti-RFP (Rockland/Biomol 1:500, catalogue no 600-401-379), rabbit anti-Ki67 (Thermo Scientific; 1:100, catalogue no RM-9106-5, clone SP6), rabbit anti-NG2 (Millipore; 1:500, catalogue no AB5320), rabbit anti-Olig2 (Millipore; 1:500, catalogue no AB9610) and rat anti-PDGFRa (BD Pharmingen; 1:200, catalogue no 558774, clone APA5). Secondary antibodies were chosen according to the primary antibodies and were coupled to Alexa Fluor 488 or FITC, Cy3, Alexa Fluor 647 or Cy5, and sections were counterstained with DAPI (Sigma Aldrich).

Western blot. SEZ culture medium was collected and concentrated by using Amicon Ultra centrifugal filters (Ultracek-10K, Millipore). Concentrated samples were then mixed with $2 \times$ sample buffer (Sigma Aldrich). SEZ cultured cells were lysed by addition of radioimmunoprecipitation assay buffer supplemented with $0.1 \%$ SDS and protease inhibitor cocktail (Complete, Roche). Protein determination of the cell extracts was done and then mixed with $2 \times$ sample buffer. All the samples were heated at $99^{\circ} \mathrm{C}$ and aliquots were subjected to SDS gel electrophoresis using $12 \%$ acrylamide gels. Immunotransference was carried out in polyvinylidene difluoride membranes (Millipore). Tris-buffered saline containing 1\% (vol./vol.) Tween-20 and $3 \%$ milk powder was employed as blocking medium and in subsequent incubations with the antibodies. Incubation with the antibodies was carried out at the following dilutions: Wnt3/3a (Cell Signalling, catalogue no 2391), 1:1,000; mouse $\alpha$-tubulin (Sigma; catalogue no T5168, clone B-5-1-2), 1:2,000. Primary antibodies were detected with horseradish peroxidase-conjugated antibodies (Invitrogen, 1:3,000, anti-mouse catalogue no G21040, and 1:3,000 anti-rabbit catalogue no G21234), and visualized by the electrochemiluminescence method (GE Healthcare).

Viral vector injections. Lentiviral constructs encoding for GFP and Wnt3 were used as described in ref. 31. Retroviral constructs were used as described in ref. 54. Injections were carried out as previously described in ref. 55. Briefly, mice were anaesthetized (ketamine ( $100 \mathrm{mg} \mathrm{kg}^{-1}$, CP Pharma) and xylazine $\left(5 \mathrm{mg} \mathrm{kg}^{-1}\right.$, Rompun, Bayer)) and injected with approximately $0.5 \mu \mathrm{l}$ of viral suspension at 0.7 (anteroposterior), 1.2 (mediolateral) and 1.6-2.1 (dorsoventral) (relative to bregma) for adult SEZ.

Wnt3a infusion. Wnt3a (R6D Systems) dissolved in artificial CSF (aCSF) or aCSF only was infused at a rate of $250 \mathrm{ng}$ per day for 7 days into the lateral ventricle of C57BL/6/J mice at the coordinates_0.2 (anteroposterior), 1 (mediolateral), 2 (dorsoventral) by osmotic minipumps $\left(1 \mu \mathrm{h}^{-1}\right.$ ALZET osmotic minipumps 2001, Sigma; Brain Infusion Kit II, Sigma). Animals were killed 4 days after Wnt3a infusion. The analysis was carried out in the contralateral side of the minipump insertion. 
Statistical analysis . Histograms show s.e.m. Statistical tests were carried out using GraphPad Prism 4.03 or MATLAB 7.11; $P$-values were calculated by $t$-test, or ANOVA with Tukey's post-test.

49. Costa, M. R., Wen, G., Lepier, A., Schroeder, T. \& Götz, M. Par-complex proteins promote proliferative progenitor divisions in the developing mouse cerebral cortex. Development 135, 11-22 (2008).

50. Eilken, H. M., Nishikawa, S. \& Schroeder, T. Continuous single-cell imaging of blood generation from haemogenic endothelium. Nature 457, 896-900 (2009).

51. Schroeder, T. Long-term single-cell imaging of mammalian stem cells. Nat. Methods 8, S30-S35 (2011).
52. Rieger, M. A., Hoppe, P. S., Smejkal, B. M., Eitelhuber, A. C. \& Schroeder, T. Hematopoietic cytokines can instruct lineage choice. Science 325, 217-218 (2009).

53. Livak, K. J. \& Schmittgen, T. D. Analysis of relative gene expression data using real-time quantitative PCR and the 2(-Delta Delta $\mathrm{C}(\mathrm{T})$ ) Method. Methods 25, 402-408 (2001).

54. Heinrich, C. et al. Directing astroglia from the cerebral cortex into subtype specific functional neurons. PLoS Biol. 8, e1000373 (2010).

55. Brill, M. S. et al. A dlx2- and pax6-dependent transcriptional code for periglomerular neuron specification in the adult olfactory bulb. J. Neurosci. 28, 6439-6452 (2008). 


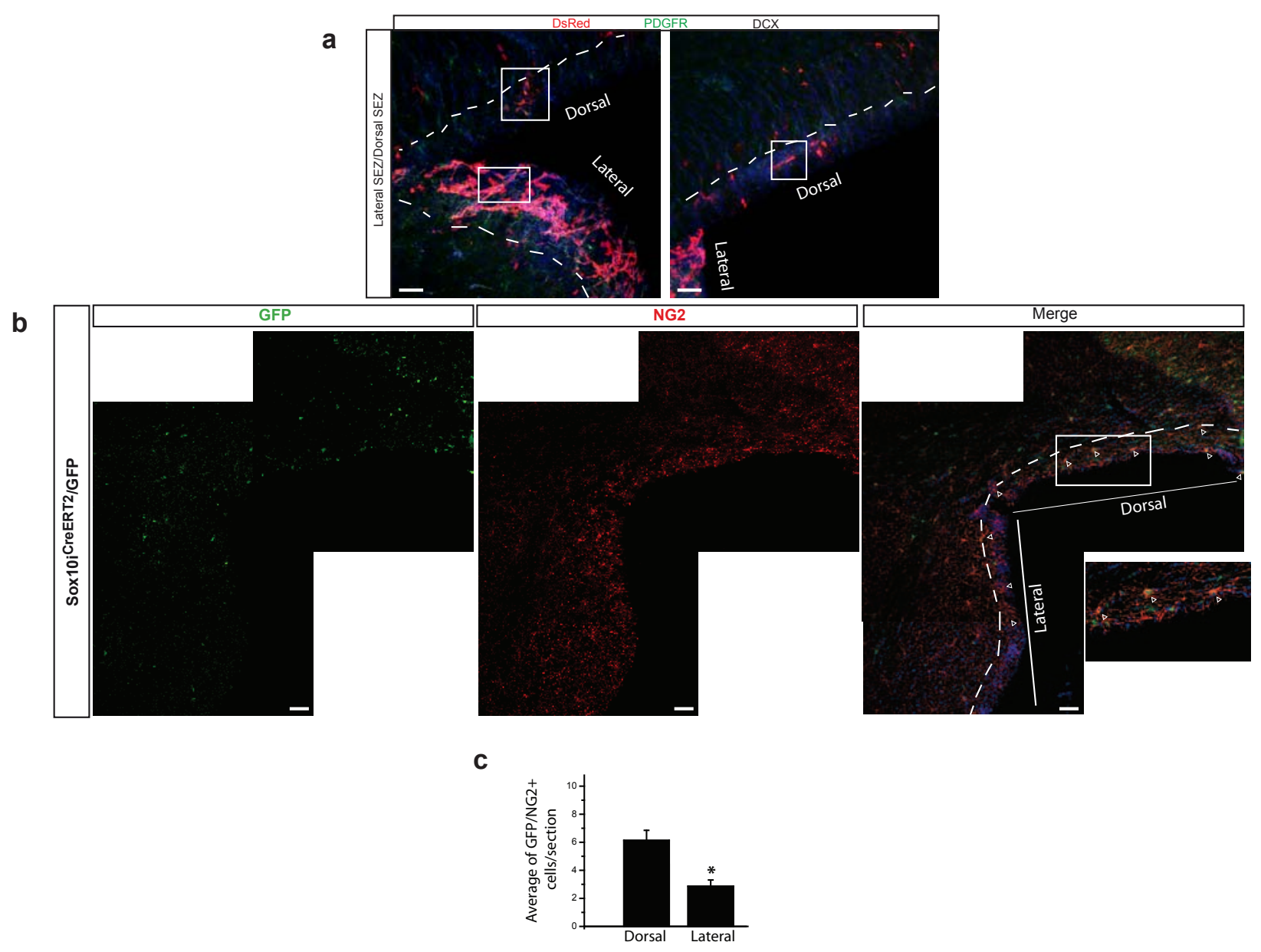

Figure S1 Oligodendroglial progenitors are present throughout the lateral and dorsal wall, but enriched in the latter. (a) Lower magnification views of the sections shown in Fig. 1d (indicated by white boxes). (b) Presence of reporter-positive (green) cells co-expressing NG2 (red) in the dorsal and lateral walls of Sox10_icreERT2/GFP mice. Note the higher frequency of oligodendroglial cells in the dorsal SEZ. (c) Quantification of the GFP and NG2 double-positive cells per section in the dorsal and lateral walls of Sox10iCreERT2/GFP mice, $t-$ Test, ${ }^{*}=p<0.05$, S.E.M, $n=8$ independent experiments). 

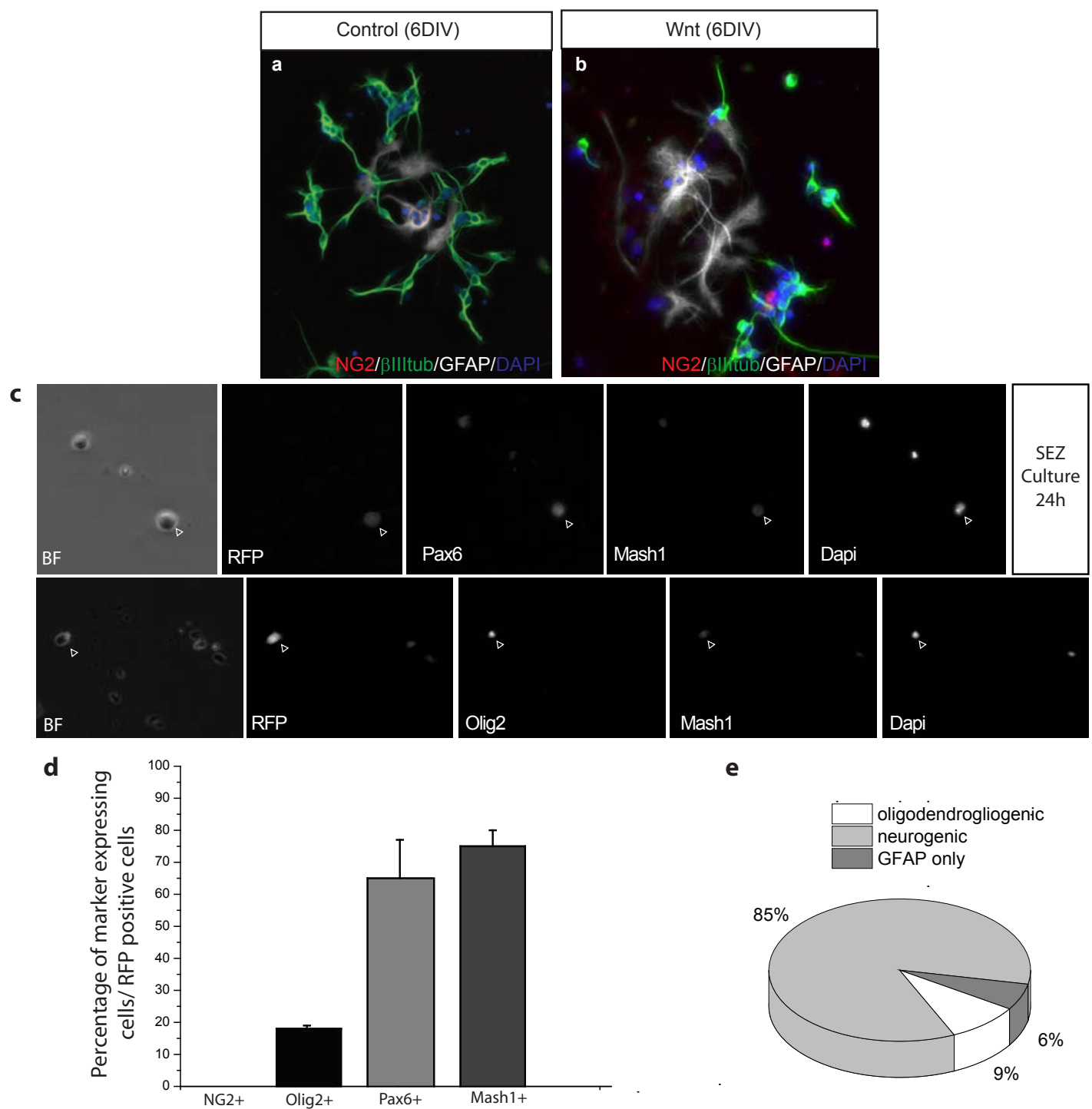

e

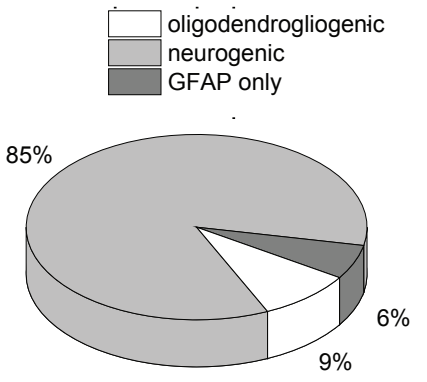

Figure S2 Post-imaging immunocytochemistry for $\beta$ III-tubulin, NG2 and GFAP expression in the neurogenic clones lineage tracked in Fig. 2a (a, control) and Fig. 4h (b, Wnt3a). (c) Phase contrast, RFP fluorescence (active GFAP promoter) and post-imaging immunocytochemistry micrographs for Pax6, Mash1, Olig2 and Dapi in adult SEZ cultures of hGFAP-RFP mice $24 \mathrm{~h}$ after continous live imaging. (d) Quantification of the percentage of populations double-positive for RFP and the following markers: (NG2, Olig2, Pax6 and Mash1) after $24 \mathrm{~h}$ in culture. (e) Quantificantion of the number of progenitors that give rise to clones belonging to the oligodendrogliogenic or neurogenic lineage, or containing only GFAP-positive cells after 7 days of continous live imaging. 
a Control lineage trees
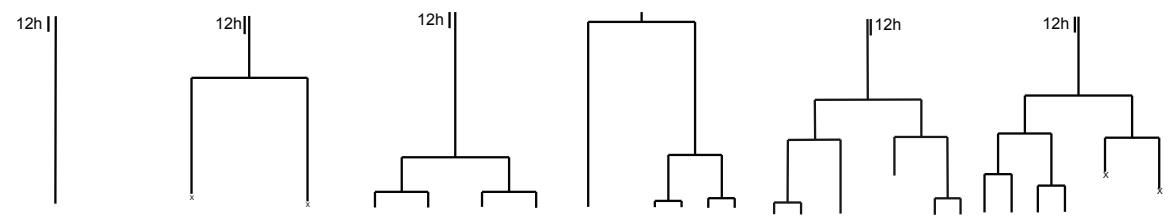

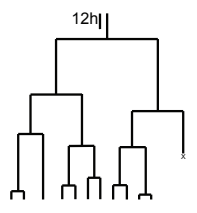

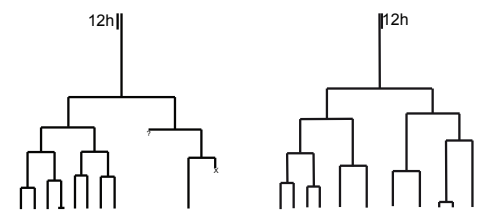

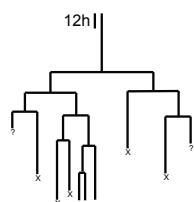

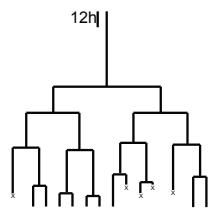<smiles>C#CCCC(CC#C)CC(CC#C)CC(C)CC(C)CC#C</smiles>

b Wnt3a treatment lineage trees<smiles>CCC(C)CC</smiles>

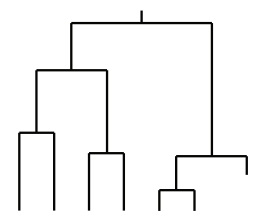<smiles>C#CCC(CCC)CCC(C)CCC</smiles>

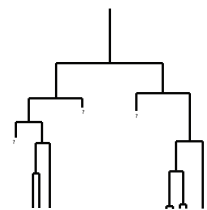<smiles>C#CC(CCC#CC)(CCCC)CC(C)(C)CC</smiles><smiles>C#CCCCC(C)CCC#C</smiles>

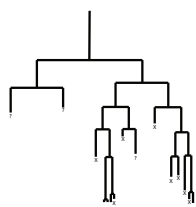

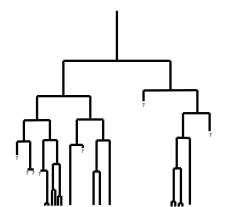

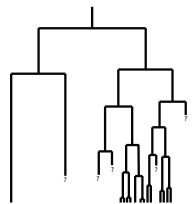

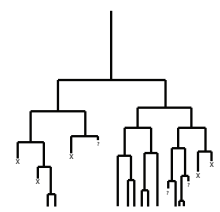

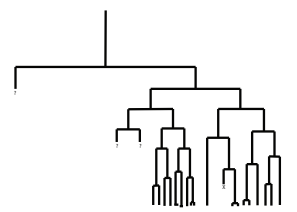

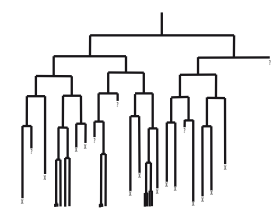

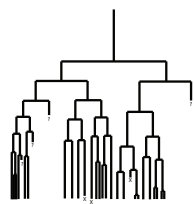
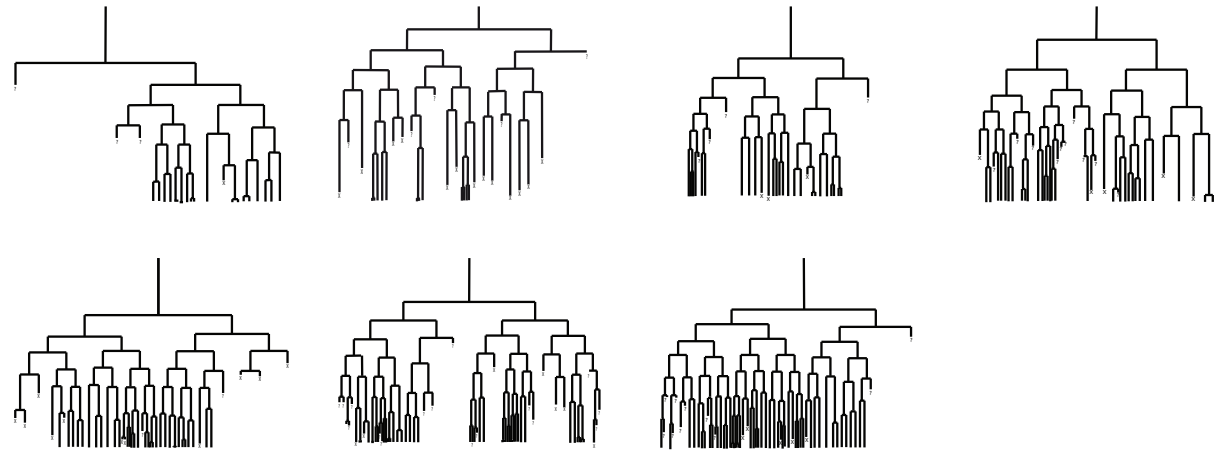

Figure S3 Summary of all oligodendrogliogenic clones tracked in a representative experiment. (a) Control culture. (b) Wnt3a-treated culture. In each case, the identity of the progeny has been determined by post-imaging ICC. 
a
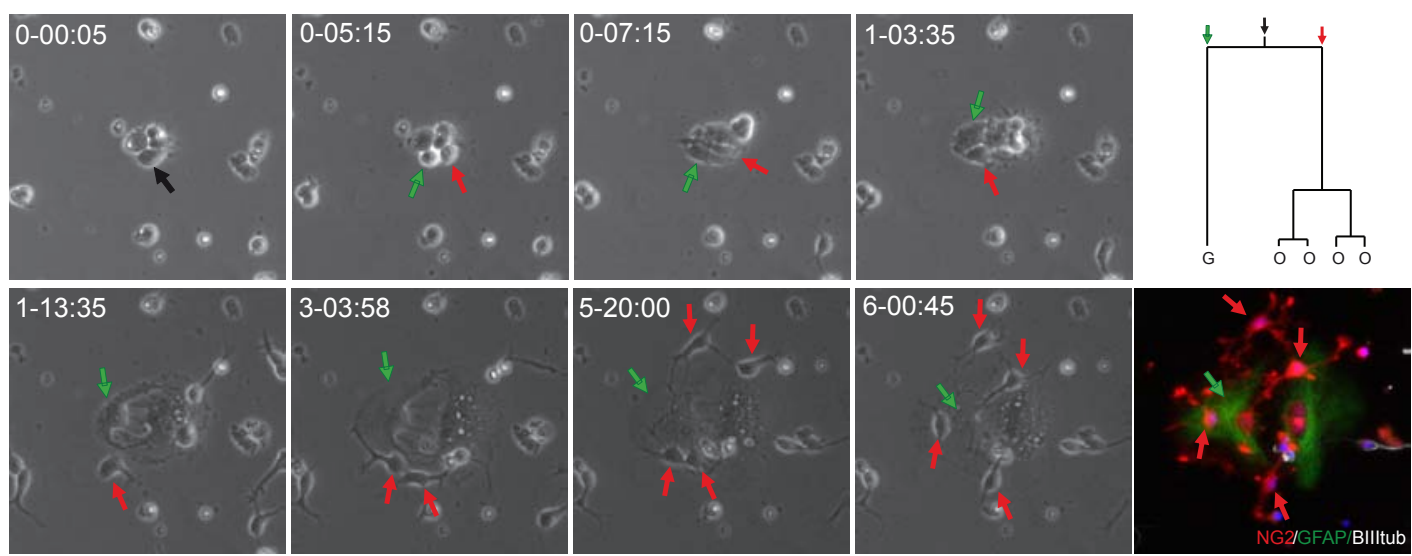

b
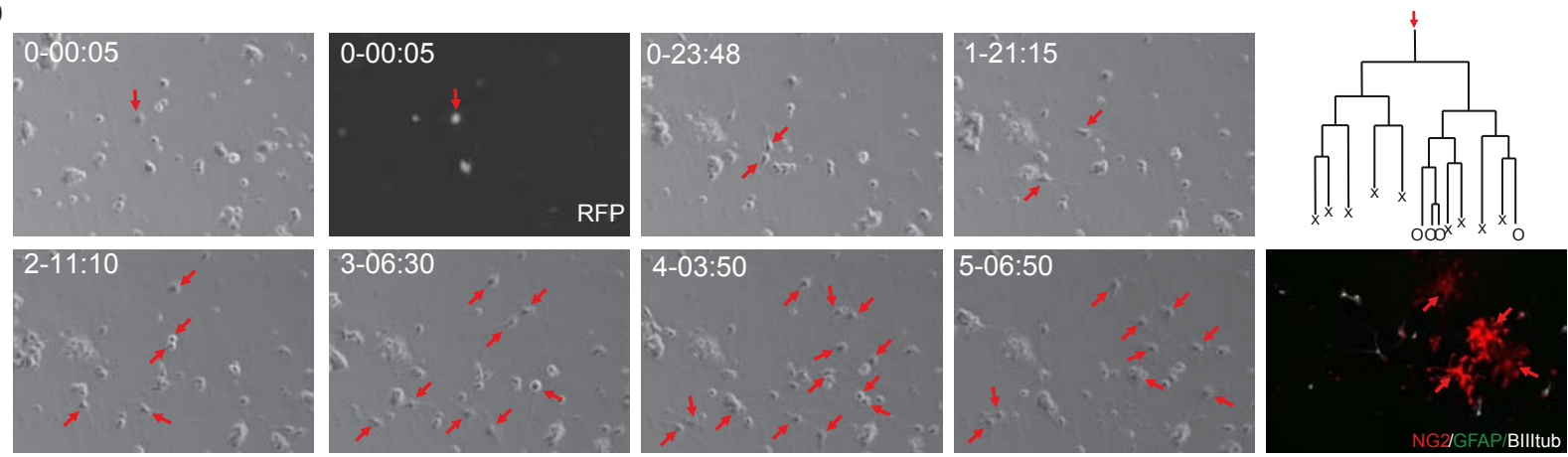

Figure S4 Examples of oligodendrogliogenic clones tracked by live imaging in cultures of the dorsal plus lateral wall of the adult SEZ under control conditions. (a) Example of an asymmetric clone generating both oligodendroglial (red arrows) and astroglial (green arrows) progeny. (b) Example of an oligodendrogliogenic clone derived from a RFP-positive founder cell in a culture derived from a hGFAP-RFP transgenic mouse. 
a
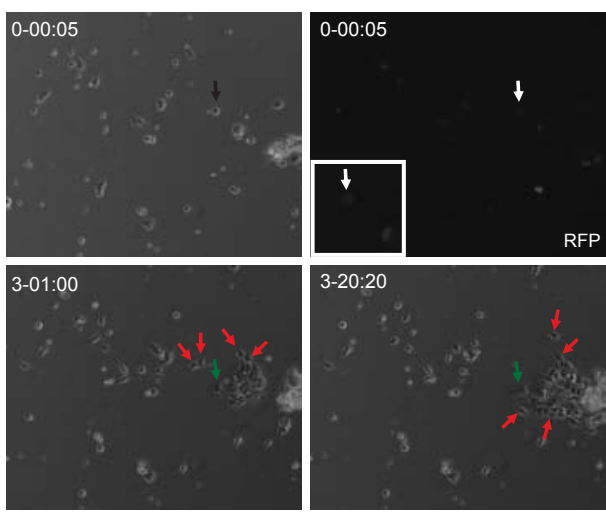

FP

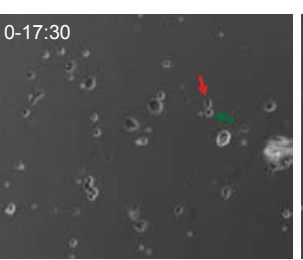

$4-13: 40$

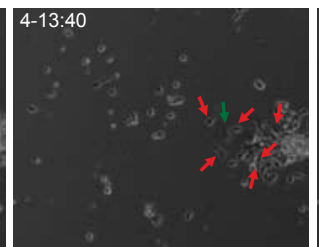

b
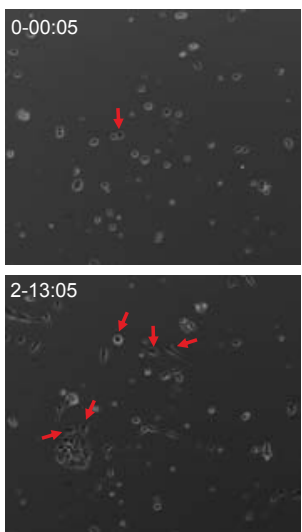
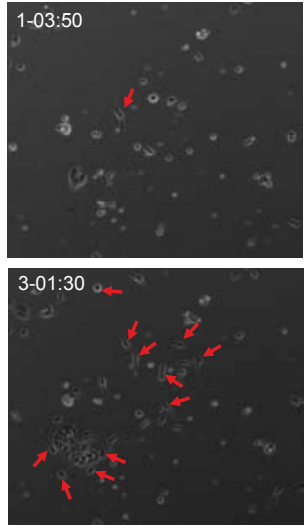
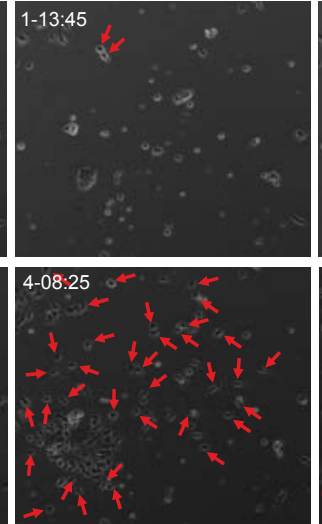
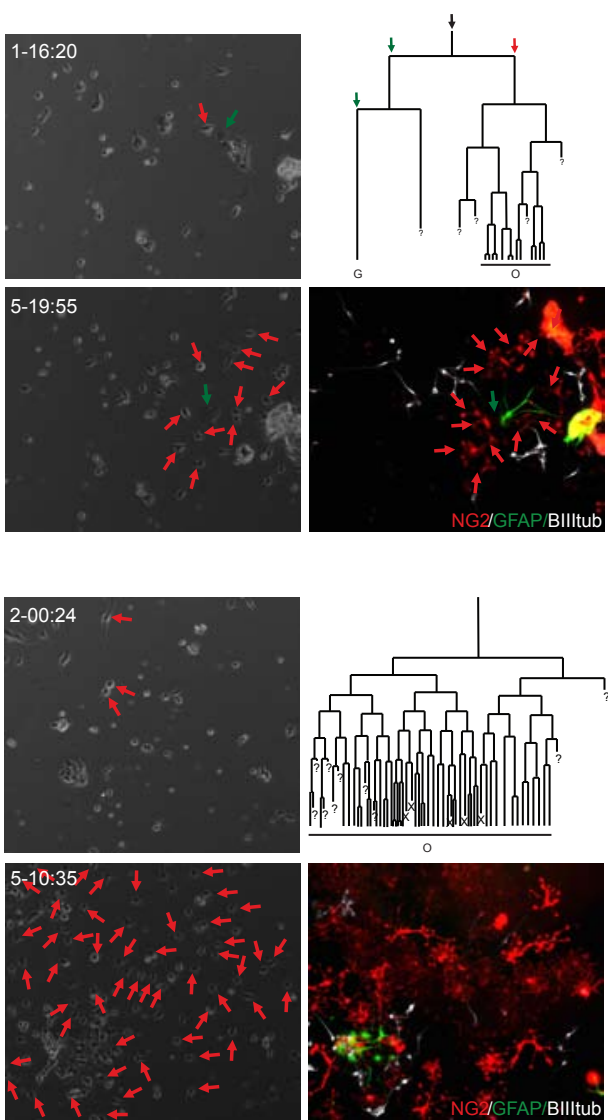

Figure S5 Examples of oligodendrogliogenic clones tracked by live imaging in cultures of the dorsal plus lateral wall of the adult SEZ following treatment with Wnt3a. (a) Example of an asymmetric clone derived from a RFP-positive founder cell in a culture derived from a hGFAP-RFP transgenic mouse generating both oligodendroglial (red arrows) and astroglial (green arrows) progeny. (b) Example of an oligodendrogliogenic clone following Wnt3a treatment exhibiting up to 8 rounds of division during the imaging period. 
a
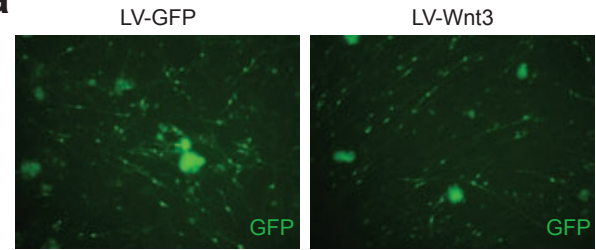

b

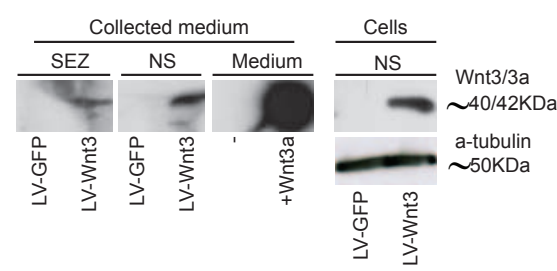

d

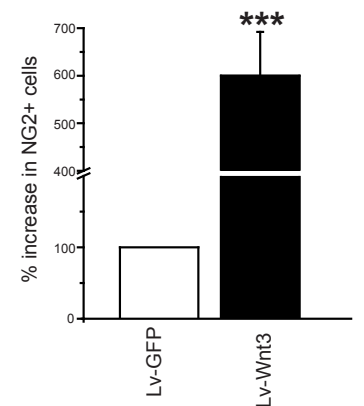

C
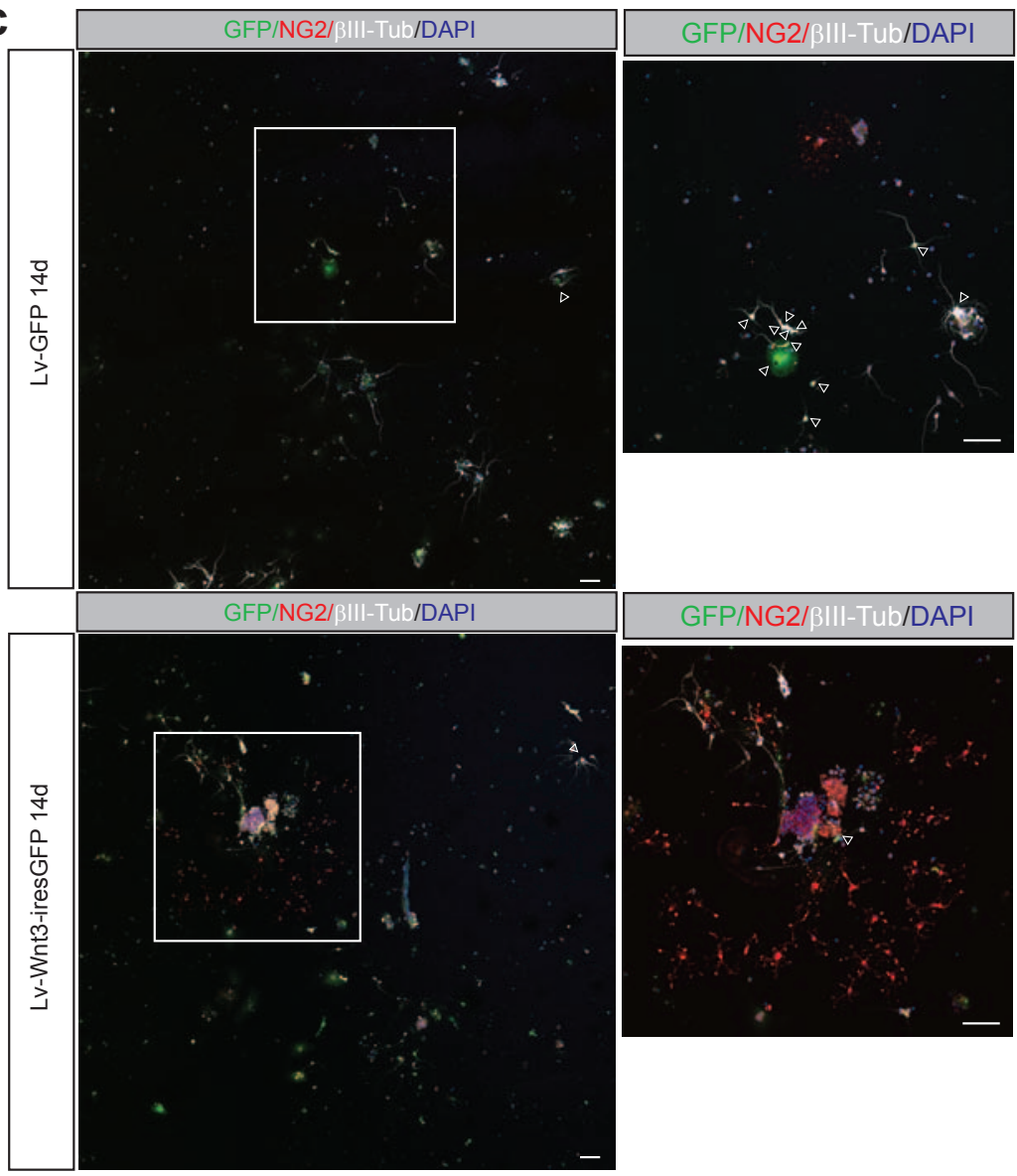

Figure S6 Lentivirus-expressed Wnt3 is biologically active. (a) Transduction of cells isolated from the adult SEZ and expanded in EGF and FGF2 with a control lentivirus (LV-GFP) or a lentivirus encoding Wnt3 (LV-Wnt3). (b) Western blot analysis for Wnt3 protein released into the medium (Collected medium) of control and Wnt3-expressing primary SEZ cultures (SEZ) and SEZ cultures expanded with EGF and FGF2 (NS). Medium containing recombinant Wnt3a was used as a positive control (Medium). Note the presence of Wnt3 protein after transduction with LV-Wnt3-iresGFP. Likewise, Wnt3 protein could be detected in the cell extract of LV-Wnt3-iresGFP transduced, but not control transduced (LV-GFP) cells. (c) Effect of lentivirus-mediated expression of Wnt3 on the number of NG2-positive oligodendroglia. Note the increase in NG2positive cells in the LV-Wnt3-iresGFP-transduced culture compared to control

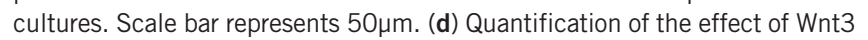
expression on the number of NG2-positive cells. Note that Wnt3 released into the medium has a similar effect as addition of recombinant Wnt3a protein (t-Test, ${ }^{* * *}=p<0.001$, S.E.M, $n=3$ independent experiments). 


\section{Supplementary Table Legends}

Supplementary Table 1 Primers used for real-time qPCR experiments.

Supplementary Table 2 Relative mRNA levels in control and Wnt3-treated cultures analyzed in Fig. 3d.

\section{Supplementary Video Legends}

Supplementary Video 1 Oligodendrogliogenic lineage progression in control cultures. The video shows the same examples as depicted in Figure $2 \mathrm{~b}, \mathrm{c}$. Clonal founder cells exhibited the hallmarks of the aNSCs such as marked cell growth prior to division and slow cell cycle. Of note, in contrast to neurogenic clones which typically remain compact through transit-amplifying divisions until reaching the neuroblast stage, oligodendrogliogenic clones display marked migratory behaviour at all stages.

Supplementary Video 2 Oligodendrogliogenic lineage progression following Wnt3a treatment. The video shows the example depicted in Figure 4a,b. The culture was prepared from the adult SEZ of a hGFAP-RFP mouse. Note the up-regulation of RFP in the clonal founder cell prior to division and the massive expansion of the clone in the presence of Wnt3a.

Supplementary Video 3 The enhancement of oligodendrogliogenesis in adult SEZ culture is not due to a switch in cell fate. The movie shows the two clones depicted in Fig. 5a. Following a control period of approximately 7 days recombinant Wnt3a was added to the culture medium addition of Wnt3a. No change was observed in the neurogenic clone (blue arrow) upon Wnt3a treatment, while the length of the cell cycle was shortened in an asymmetrically dividing oligodendrogliogenic clone (red arrow). Note that the astroglial progeny did not respond to Wnt3a stimulation. 